Algebraic $\&$ Geometric Topology

Volume 3 (2003) 207-234

Published: 26 February 2003

ATG

\title{
Limit points of lines of minima in Thurston's boundary of Teichmüller space
}

\author{
Raquel Díaz \\ Caroline Series
}

\begin{abstract}
Given two measured laminations $\mu$ and $\nu$ in a hyperbolic surface which fill up the surface, Kerckhoff [8] defines an associated line of minima along which convex combinations of the length functions of $\mu$ and $\nu$ are minimised. This is a line in Teichmüller space which can be thought as analogous to the geodesic in hyperbolic space determined by two points at infinity. We show that when $\mu$ is uniquely ergodic, this line converges to the projective lamination $[\mu]$, but that when $\mu$ is rational, the line converges not to $[\mu]$, but rather to the barycentre of the support of $\mu$. Similar results on the behaviour of Teichmüller geodesics have been proved by Masur [9].
\end{abstract}

AMS Classification 20H10; 32G15

Keywords Teichmüller space, Thurston boundary, measured geodesic lamination, Kerckhoff line of minima

\section{Introduction}

Let $S$ be a surface of hyperbolic type, and denote its Teichmüller space by Teich $(S)$. Given a measured geodesic lamination $\mu$ on $S$ (see Section 2 for definitions), there is a function $l_{\mu}$ : $\operatorname{Teich}(S) \rightarrow \mathbb{R}^{+}$which associates to each $\rho \in \operatorname{Teich}(S)$ the hyperbolic length $l_{\mu}(\rho)$ of $\mu$ in the hyperbolic structure $\rho$. If $\mu, \nu$ are two measured laminations which fill up the surface, Kerckhoff [8] proved that for any number $s \in(0,1)$, the function $F_{s}=(1-s) l_{\mu}+s l_{\nu}$ has a global minimum at a unique point $m_{s} \in \operatorname{Teich}(S)$. The set of all these minima, when $s$ varies in the interval $(0,1)$, is called a line of minima $\mathcal{L}_{\mu, \nu}$.

The Teichmüller space of a surface is topologically a ball which, as shown by Thurston, can be compactified by the space $\mathrm{P} \mathcal{M L}$ of projective measured laminations on $S$. Various analogies between Teichmüller space and hyperbolic space have been studied, for example earthquake paths in Teichmüller space are analogous to horocycles in hyperbolic space. In [8], Kerckhoff studied some 
properties of the lines of minima analogous to properties of geodesics in hyperbolic space. For example, two projective measured laminations determine a unique line of minima, in analogy to the fact that two different points in the boundary of hyperbolic space determine a unique geodesic. He warns, however, that lines of minima do not always converge to the point corresponding to $\mu$ in Thurston's compactification of Teich $(S)$, mentioning that examples can be constructed by taking $\mu$ rational (that is, such that its support consists entirely of closed leaves). In this paper we make this explicit by showing that any line of minima $\mathcal{L}_{\mu, \nu}$ where $\mu=\sum a_{i} \alpha_{i}$, with $a_{i}>0$, converges to the projective lamination $\left[\sum \alpha_{i}\right]$, rather than to $\left[\sum a_{i} \alpha_{i}\right]$.

Theorem 1.1 Let $\mu=\sum_{i=1}^{N} a_{i} \alpha_{i}$ be a rational measured lamination (that is, $\alpha_{i}$ is a collection of disjoint simple closed curves on $S$ and $a_{i}>0$ for all $i)$ and $\nu$ any measured lamination so that $\mu, \nu$ fill up the surface. For any $0<s<1$, consider the function $F_{s}: \operatorname{Teich}(S) \rightarrow \mathbb{R}$ defined by $F_{s}(\rho)=$ $(1-s) l_{\mu}(\rho)+s l_{\nu}(\rho)$, and denote its unique minimum by $m_{s}$. Then

$$
\lim _{s \rightarrow 0} m_{s}=\left[\alpha_{1}+\cdots+\alpha_{N}\right] \in \mathrm{P} \mathcal{M} \mathcal{L} .
$$

By contrast, if $\mu$ is uniquely ergodic and maximal (see Section 2 for the definition), we prove:

Theorem 1.2 Let $\mu$ and $\nu$ be two measured laminations which fill up the surface and such that $\mu$ is uniquely ergodic and maximal. With $m_{s}$ as above,

$$
\lim _{s \rightarrow 0} m_{s}=[\mu] \in \mathrm{P} \mathcal{M} \mathcal{L} .
$$

Exactly similar results have been proved by Masur [9] for Teichmüller geodesics. In this case, a geodesic ray is determined by a base surface $\rho$ and a quadratic differential $\phi$ on $\rho$. Roughly speaking, the end of this ray depends on the horizontal foliation $F$ of $\phi$. Masur shows that if $F$ is a Jenkins-Strebel differential, that is, if its horizontal foliation has closed leaves, then the associated ray converges in the Thurston boundary to the barycentre of the leaves (the foliation with the same closed leaves all of whose cylinders have unit height), while if $F$ is uniquely ergodic and every leaf (apart from saddle connections) is dense in $S$, it converges to the boundary point defined by $F$.

Our interest in lines of minima arose from the study of the space $\mathcal{Q F}(S)$ of quasifuchsian groups associated to a surface $S$. The pleating plane determined by a pair of projective measured laminations is the set of quasifuchsian groups whose convex hull boundary is bent along the given laminations with bending 
measure in the given classes. It is shown in [14], see also [15], that if $\mu, \nu$ are measured laminations, then the closure of their pleating plane meets fuchsian space exactly in the line of minima $\mathcal{L}_{\mu, \nu}$.

From this point of view, it is often more natural to look at the collection of all groups whose convex hulls are bent along a specified set of closed curves. That is, we forget the proportions between the bending angles given by the measured lamination $\mu$ and look only at its support. This led us in [3] to study the simplex of minima determined by two systems of disjoint simple curves on the twice punctured torus, where direct calculation of some special examples led to our results here.

The simplex of minima $\mathcal{S}_{\mathcal{A}, \mathcal{B}}$ associated to systems of disjoint simple curves $\mathcal{A}=\left\{\alpha_{1}, \ldots, \alpha_{N}\right\}$ and $\mathcal{B}=\left\{\beta_{1}, \ldots, \beta_{M}\right\}$ which fill up the surface, is the union of lines of minima $\mathcal{L}_{\mu, \nu}$, where $\mu, \nu \in \mathcal{M L}(S)$ are strictly positive linear combinations of $\left\{\alpha_{i}\right\}$ and $\left\{\beta_{i}\right\}$, respectively. We can regard $\mathcal{S}_{\mathcal{A}, \mathcal{B}}$ as the image of the affine simplex $S_{\mathcal{A}, \mathcal{B}}$ in $\mathbb{R}^{N+M-1}$ spanned by independent points $A_{1}, \ldots, A_{N}, B_{1}, \ldots, B_{M}$, under the map $\Phi$ which sends the point (1$s)\left(\sum_{i} a_{i} A_{i}\right)+s\left(\sum_{j} b_{j} B_{j}\right)$ (with $0<s, a_{i}, b_{j}<1, \sum a_{i}=1, \sum b_{j}=1$ ) to the unique minimum of the function $(1-s)\left(\sum_{i} a_{i} l_{\alpha_{i}}\right)+s\left(\sum_{j} b_{j} l_{\beta_{j}}\right)$.

As observed in [3], the methods of [8] show that the map $\Phi$ is continuous and proper. It may or may not be a homeomorphism onto its image; in [3] we give a necessary and sufficient condition and show by example that both cases occur. The map extends continuously to the faces of $S_{\mathcal{A}, \mathcal{B}}$ which correspond to curves $\left\{\alpha_{i_{1}}, \ldots, \alpha_{i_{k}}\right\},\left\{\beta_{j_{1}}, \ldots, \beta_{j_{l}}\right\}$ that still fill up the surface. Nevertheless, as a consequence of Theorem 1.1, $\Phi$ does not necessarily extend to a function from the closure of $S_{\mathcal{A}, \mathcal{B}}$ into the Thurston boundary.

Corollary 1.3 Let $\mathcal{A}, \mathcal{B}$ be as above and suppose that $\left\{\alpha_{1}, \ldots, \alpha_{N-1}\right\}$ and $\mathcal{B}=\left\{\beta_{1}, \ldots, \beta_{M}\right\}$ also fill up $S$. Then, the map $\Phi: S_{\mathcal{A}, \mathcal{B}} \rightarrow \operatorname{Teich}(S)$ does not extend continuously to a function $\overline{S_{\mathcal{A}, \mathcal{B}}} \rightarrow \operatorname{Teich}(S) \cup \operatorname{P\mathcal {ML}}(S)$.

Proof Let $\left\{x_{n}\right\}$ be a sequence of points in $S_{\mathcal{A}, \mathcal{B}}$, and $\left\{y_{n}\right\}$ another sequence in the face spanned by $A_{1}, \ldots, A_{N-1}, B_{1}, \ldots, B_{M}$, both converging to $\left(A_{1}+\right.$ $\left.\cdots+A_{N-1}\right) /(N-1)$. Then, by Theorem 1.1, $\Phi\left(x_{n}\right)$ converges to $\left[\alpha_{1}+\cdots+\alpha_{N}\right]$ while $\Phi\left(y_{n}\right)$ converges to $\left[\alpha_{1}+\cdots+\alpha_{N-1}\right]$.

We remark that examples of curve systems as in the corollary are easy to construct. 
The paper is organised as follows. The main work is in proving Theorem 1.1. In Section 2 we recall background and give the (easy) proof of Theorem 1.2. In Section 3 we study an example which illustrates Theorem 1.1 and its proof. The general proof is easier when $\alpha_{1}, \ldots, \alpha_{N}$ is a pants decomposition. We work this case in Sections 4 and 5, leaving the non-pants decomposition case for Section 6.

The first author would like to acknowledge partial support from MCYT grant BFM2000-0621 and UCM grant PR52/00-8862, and the second support from an EPSRC Senior Research Fellowship.

\section{Background}

We take the Teichmüller space Teich $(S)$ of a surface $S$ of hyperbolic type to be the set of faithful and discrete representations $\rho: \pi_{1}(S) \rightarrow P S L(2, \mathbb{R})$ which take loops around punctures to parabolic elements, up to conjugation by elements of $P S L(2, \mathbb{R})$. An element $\rho$ of Teich $(S)$ can be regarded as a marked hyperbolic structure on $S$. The space Teich $(S)$ is topologically a ball of dimension $2(3 g-3+b)$, where $g$ is the genus and $b$ the number of punctures of $S$. A pants decomposition of $S$ is a set of disjoint simple closed curves, $\left\{\alpha_{1}, \ldots, \alpha_{N}\right\}$ which decompose the surface into pairs of pants $(N=3 g-3+b)$. Given a pants decomposition $\left\{\alpha_{1}, \ldots, \alpha_{N}\right\}$ on $S$, the Fenchel-Nielsen coordinates give a global parameterization of Teich $(S)$. Given a marked hyperbolic structure on $S$, these coordinates consist of the lengths $l_{\alpha_{i}}$ of the geodesics representing the curves $\alpha_{i}$, and the twist parameters $t_{\alpha_{i}}$. The lengths $l_{\alpha_{i}}$ determine uniquely the geometry on each pair of pants, while the twist parameters are real numbers determining the way these pairs of pants are glued together to build up the hyperbolic surface. We need to specify a set of base points in Teich $(S)$, namely a subset of Teich $(S)$ where the twist parameters are all equal to zero. This can be done by choosing a set of curves $\left\{\delta_{i}\right\}$ dual to the $\left\{\alpha_{i}\right\}$, in the sense that each $\delta_{i}$ intersects $\alpha_{i}$ either once or twice and is disjoint from $\alpha_{j}$ for all $j \neq i$. For each fixed set of values of $l_{\alpha_{i}}$, the base point is then the marked hyperbolic structure in which each $\delta_{i}$ is orthogonal to $\alpha_{i}$, when they intersect once, or in which the two intersection angles (measured from $\delta_{i}$ to $\alpha_{i}$ ) sum to $\pi$, when they intersect twice.

A geodesic lamination in a hyperbolic surface $\rho$ is a closed subset of $\rho$ which is disjoint union of simple geodesics, called its leaves. A geodesic lamination is measured if it carries a transverse invariant measure (for details, see for example $[4,10]$ and the appendix to [11]). The space $\mathcal{M L}$ of measured laminations 
is given the weak topology. If $\mu \in \mathcal{M L}$, then $|\mu|$ will denote its underlying support. To exclude trivial cases, we assume that each leaf $l$ of $|\mu|$ is a density point of $\mu$, meaning that any open interval transverse to $l$ has positive $\mu$-measure. In this paper, we shall mainly use rational measured laminations, denoted by $\sum_{i} a_{i} \alpha_{i}$, where $a_{i} \in \mathbb{R}^{+}$and $\alpha_{i}$ are disjoint simple closed geodesics. This measured lamination assigns mass $a_{i}$ to each intersection of a transverse arc with $\alpha_{i}$. The length of a rational lamination $\sum_{i} a_{i} \alpha_{i}$ on a hyperbolic surface $\rho$ is defined to be $\sum_{i} a_{i} l_{\alpha_{i}}(\rho)$, where $l_{\alpha_{i}}(\rho)$ is the length of $\alpha_{i}$ at $\rho$. Rational measured laminations are dense in $\mathcal{M L}$ and the length of a measured lamination can be defined as the limit of the lengths of approximating rational measured laminations, see [6]. This construction appears to depend on $\rho$, however a homeomorphism between hyperbolic surfaces transfers geodesic laminations canonically from the first surface to the second. Thus, given a measured lamination $\mu$, there is a map $l_{\mu}: \operatorname{Teich}(S) \rightarrow \mathbb{R}^{+}$which assigns to each point $\rho \in \operatorname{Teich}(S)$ the length $l_{\mu}(\rho)$ of $\mu$ on the hyperbolic structure $\rho$. The map $l_{\mu}$ is real analytic with respect to the real analytic structure of Teich $(S)$, see [6] Corollary 2.2.

Two measured laminations are equivalent if they have the same underlying support and proportional transverse measures. The equivalence class of a measured lamination $\mu$ is called a projective measured lamination and is denoted by $[\mu]$. A measured lamination is maximal if its support is not contained in the support of any other measured lamination. A lamination is uniquely ergodic if every lamination with the same support is in the same projective equivalence class. (Thus the lamination $\sum_{i} a_{i} \alpha_{i}$ is uniquely ergodic if and only if the sum contains exactly one term.) The geometric intersection number $i\left(\gamma, \gamma^{\prime}\right)$ of two simple closed geodesics is the number of points in their intersection. This number extends by bilinearity and continuity to the intersection number of measured laminations, see $[12,6,2]$. The following characterisation of uniquely ergodic laminations is needed in the proof of Theorem 1.2.

Lemma 2.1 A lamination $\mu \in \mathcal{M L}$ is uniquely ergodic and maximal if and only if, for all $\nu \in \mathcal{M L}, i(\mu, \nu)=0$ implies $\nu \in[\mu]$.

Proof If $i(\mu, \nu)=0$ implies $\nu \in[\mu]$, it is easy to see that $\mu$ must be uniquely ergodic and maximal. The converse follows using the definition of intersection number as the integral over $S$ of the product measure $\mu \times \nu$; see for example [6]. Since we are assuming $\mu$ is uniquely ergodic, it is enough to show that the supports of $\mu$ and $\nu$ are the same.

Let $\omega$ be the lamination consisting of leaves (if any) which are common to $|\mu|$ and $|\nu|$. Let $\mu_{\omega}$ and $\nu_{\omega}$ denote the restrictions of $\mu$ and $\nu$ to $\omega$. Clearly $\omega$ 
is closed, and hence (using the decomposition of laminations into finitely many minimal components, see for example [1],[11]), one can write $\mu=\mu_{\omega}+\mu^{\prime}$, $\nu=\nu_{\omega}+\nu^{\prime}$ where $\mu^{\prime}, \nu^{\prime}$ are (measured) laminations disjoint from $\omega$ such that every leaf of $\mu^{\prime}$ is transverse to every leaf of $\nu^{\prime}$. Since $\mu$ is uniquely ergodic, one or other of $\mu^{\prime}$ or $\mu_{\omega}$ is zero. In the former case, maximality of $\mu$ forces $\nu^{\prime}=0$, and we are done.

Thus we may assume that every leaf of $|\mu|$ intersects every leaf of $|\nu|$ transversally; let $X$ be the set of intersection points of these leaves. Cover $X$ by small disjoint open 'rectangles' $R_{i}$, each with two 'horizontal' and two 'vertical' sides, in such a way that $|\mu| \cap R$ consists entirely of arcs with endpoints on the horizontal sides and similarly for $|\nu| \cap R$ replacing horizontal by vertical. Put a product measure on $R$ by using the transverse measure $\mu$ on 'horizontal' arcs and $\nu$ on 'vertical' ones. Then $i(\mu, \nu)=\sum_{i} \int_{R_{i}} d \mu \times d \nu$. Our assumption that each leaf of $|\mu|$ and $|\nu|$ is a density point means that the contribution to $i(\mu, \nu)$ is non-zero whenever $R \cap X$ is non-empty. Thus $i(\mu, \nu)=0$ implies that $X=\emptyset$. Since $\mu$ is maximal, every leaf of $|\nu|$ either coincides with or intersects some leaf of $|\mu|$, and we conclude that the leaves of $|\nu|$ and $|\mu|$ coincide as before.

There is a similar characterisation of uniquely ergodic foliations due to Rees [12], see also [9] Lemma 2, in which the assumption that $\mu$ is maximal is replaced by the assumption that every leaf, other than saddle connections, is dense. (Notice that the above proof shows that a uniquely ergodic lamination is also minimal, in the sense that every leaf is dense in the whole lamination.)

\subsection{The Thurston Boundary}

We denote the set of all non-zero projective measured laminations on $S$ by $\operatorname{PML}(S)$. Thurston has shown that $\operatorname{P\mathcal {ML}}(S)$ compactifies Teich $(S)$ so that $\operatorname{Teich}(S) \cup \operatorname{P} \mathcal{M L}(S)$ is homeomorphic to a closed ball. We explain this briefly; for details see [4]. A sequence $\left\{\rho_{n}\right\} \subset \operatorname{Teich}(S)$ converges to $[\mu] \in \mathrm{P} \mathcal{M L}$ if the lengths of simple closed geodesics on $\rho_{n}$ converge projectively to their intersection numbers with $\mu$; more precisely, if there exists a sequence $\left\{c_{n}\right\}$ converging to infinity, so that $l_{\gamma}\left(\rho_{n}\right) / c_{n} \rightarrow i(\gamma, \mu)$, for any simple closed geodesic $\gamma$. The following lemma summarises the consequences of this definition we shall need.

Lemma 2.2 Let $\alpha_{1}, \ldots, \alpha_{N}$ be a pants decomposition on $S$ and let $\left\{\rho_{n}\right\} \subset$ $\operatorname{Teich}(S)$ so that $\rho_{n} \rightarrow[\mu] \in \operatorname{P} \mathcal{M L}(S)$. Then:

(a) if $\nu \in \mathcal{M L}$ with $i(\mu, \nu) \neq 0$ then $l_{\nu}\left(\rho_{n}\right) \rightarrow \infty$, 
(b) if $l_{\alpha_{i}}\left(\rho_{n}\right)$ is bounded for all $i=1, \ldots, N$, then there exist $a_{1}, \ldots, a_{N} \geq 0$ so that $[\mu]=\left[a_{1} \alpha_{1}+\cdots+a_{N} \alpha_{N}\right]$.

The proofs are immediate from the definitions. Part (b) gives a sufficient condition for convergence to a rational lamination $\left[\Sigma_{i} a_{i} \alpha_{i}\right]$, when the $\left\{\alpha_{i}\right\}$ is a pants decomposition. To compute the coefficients $a_{i}$ we take another system of curves $\left\{\delta_{i}\right\}$ dual to the $\left\{a_{i}\right\}$. From the definition,

$$
\frac{l_{\delta_{j}}\left(\rho_{n}\right)}{l_{\delta_{k}}\left(\rho_{n}\right)} \rightarrow \frac{i\left(\sum a_{i} \alpha_{i}, \delta_{j}\right)}{i\left(\sum a_{i} \alpha_{i}, \delta_{k}\right)}=\frac{a_{j} i\left(\alpha_{j}, \delta_{j}\right)}{a_{k} i\left(\alpha_{k}, \delta_{k}\right)},
$$

and we know that $i\left(\alpha_{i}, \delta_{i}\right)$ is either 1 or 2 , so this gives the proportion $a_{j} / a_{k}$.

\section{$2.2 \quad$ Lines of minima}

Two measured laminations $\mu, \nu$ are said to fill up a surface $S$ if for any other lamination $\eta$ we have $i(\mu, \eta)+i(\nu, \eta) \neq 0$. It is proved in [8] that for any two such laminations, the function $l_{\mu}(\rho)+l_{\nu}(\rho)$ has a unique minimum on $\operatorname{Teich}(S)$. Thus $\mu$ and $\nu$ determine the line of minima $\mathcal{L}_{\mu, \nu}$, namely the set of points $m_{s} \in \operatorname{Teich}(S)$ at which the function $F_{s}(\rho)=(1-s) l_{\mu}(\rho)+s l_{\nu}(\rho)$ reaches its minimum as $s$ varies in $(0,1)$.

Given this definition, we can immediately prove Theorem 1.2.

Proof of Theorem 1.2 Observe that $l_{\mu}\left(m_{s}\right)$ is bounded as $s \rightarrow 0$, because $l_{\mu} \leq 2\left((1-s) l_{\mu}+s l_{\nu}\right)=2 F_{s}$ for $s<1 / 2$ and $F_{s}\left(m_{s}\right) \leq F_{s}\left(\rho_{0}\right)$ where $\rho_{0}$ is some arbitrary point in Teich $(S)$. By compactness of Teich $(S) \cup \mathrm{P} \mathcal{M L}$, we can choose some sequence $s_{n} \rightarrow 0$ for which $m_{s_{n}}$ is convergent. Moreover, it is proved in [8] that the map $s \rightarrow m_{s}$ is proper, and so $m_{s_{n}} \rightarrow[\eta] \in \mathrm{P} \mathcal{M L}$. By Lemma 2.2 (a) we have that $i(\mu, \eta)=0$ and from Lemma 2.1 we deduce that $[\eta]=[\mu]$. The result follows.

We now turn to the more interesting rational case. In general, the minimum $m_{s}$ is in fact the unique critical point of $F_{s}$, so a point $p \in \mathcal{L}_{\mu, \nu}$ if and only if the 1 -form $\mathrm{d} F_{s}=(1-s) \mathrm{d} l_{\mu}+s \mathrm{~d} l_{\nu}$ vanishes at $p$ for some $s$. If $\mu=\sum_{i}^{N} a_{i} \alpha_{i}$ is rational and $\left\{\alpha_{1}, \ldots, \alpha_{N}\right\}$ is a pants decomposition, this enables us to find equations for $\mathcal{L}_{\mu, \nu}$. In fact, applying $\mathrm{d} F_{s}=(1-s) \sum_{i} a_{i} \mathrm{~d} l_{\alpha_{i}}+s \mathrm{~d} l_{\nu}$ to the tangent vectors $\frac{\partial}{\partial t_{\alpha_{i}}}$, we get

$$
\frac{\partial l_{\nu}}{\partial t_{\alpha_{i}}}=0, \quad \text { for all } i=1, \ldots, n .
$$


Similarly, applying $\mathrm{d} F_{s}$ to the tangent vectors $\frac{\partial}{\partial l_{\alpha_{i}}}$, we get $\partial l_{\nu} / \partial l_{\alpha_{i}}=-a_{i}(1-$ $s) / s$, so that the line of minima satisfies the equations

$$
\frac{1}{a_{i}} \frac{\partial l_{\nu}}{\partial l_{\alpha_{i}}}=\frac{1}{a_{j}} \frac{\partial l_{\nu}}{\partial l_{\alpha_{j}}} \text { for all } i, j
$$

Since $\frac{\partial}{\partial l_{\alpha_{i}}}, \frac{\partial}{\partial t_{\alpha_{i}}}$ form a basis of tangent vectors ([6] Proposition 2.6), the equations (1) and (2) completely determine $\mathcal{L}_{\mu, \nu}$.

\section{Example}

Let $S=S_{1,2}$ be the twice punctured torus. Consider two disjoint, nondisconnecting simple closed curves $\alpha_{1}$ and $\alpha_{2}$, and let $\beta$ be a simple closed curve intersecting each of $\alpha_{1}$ and $\alpha_{2}$ once. For positive numbers $a_{1}, a_{2}$, denote by $\mu$ the measured lamination $a_{1} \alpha_{1}+a_{2} \alpha_{2}$. (When $S$ has a hyperbolic structure and $\gamma \in \pi_{1}(S)$, we abuse notation by using $\gamma$ to mean also the unique geodesic in the homotopy class of $\gamma$.) We shall compute the equation of the line of minima $\mathcal{L}_{\mu, \beta}$, in terms of Fenchel-Nielsen coordinates relative to the pants decomposition $\left\{\alpha_{1}, \alpha_{2}\right\}$ and dual curves $\left\{\delta_{1}, \delta_{2}\right\}$ (see Figure 1), and we shall show that this line converges in Thurston's compactification to $\left[\alpha_{1}+\alpha_{2}\right]$.

As explained above, the line of minima $\mathcal{L}_{\mu, \beta}$ is determined by the equations

$$
\frac{\partial l_{\beta}}{\partial t_{\alpha_{1}}}=0, \quad \frac{\partial l_{\beta}}{\partial t_{\alpha_{2}}}=0 \quad \text { and } \quad \frac{1}{a_{1}} \frac{\partial l_{\beta}}{\partial l_{\alpha_{1}}}=\frac{1}{a_{2}} \frac{\partial l_{\beta}}{\partial l_{\alpha_{2}}} .
$$

For two simple closed geodesics $\alpha, \beta$, Kerckhoff's derivative formula [7] states

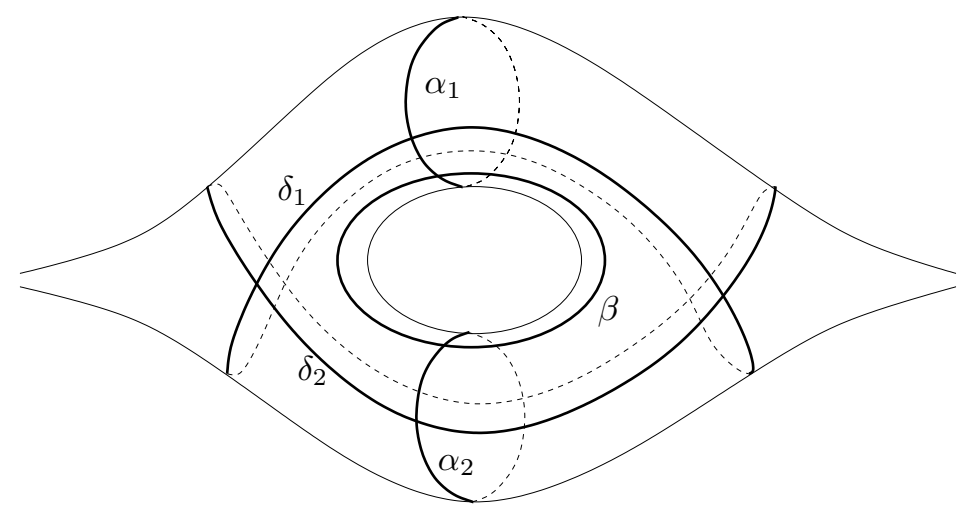

Figure 1: The curves $\alpha_{1}, \alpha_{2}$ and $\beta$ on a twice punctured torus

Algebraic 83 Geometric Topology, Volume 3 (2003) 
that $\left.\left(\partial l_{\beta} / \partial t_{\alpha}\right)\right|_{\rho}=\sum \cos \psi_{i}(\rho)$, where $\psi_{i}$ are the intersection angles from $\beta$ to $\alpha$ at each intersection point. Thus the first two equations mean that at a point in the line of minima the geodesic $\beta$ is orthogonal to $\alpha_{1}$ and $\alpha_{2}$. Let $P, P^{\prime}$ be the two pairs of pants into which $\left\{\alpha_{1}, \alpha_{2}\right\}$ split $S$; denote by $H_{12}, H_{11}$ the perpendicular segments in $P$ from the geodesic $\alpha_{1}$ to $\alpha_{2}$ and from $\alpha_{1}$ to itself, respectively; and denote by $H_{12}^{\prime}, H_{11}^{\prime}$ the analogous perpendiculars in $P^{\prime}$. Since $\beta$ is orthogonal to $\alpha_{1}$ and $\alpha_{2}, P$ and $P^{\prime}$ are glued so that the segments $H_{12}$ and $H_{12}^{\prime}$ match up. Since $P, P^{\prime}$ are isometric (each is determined by the lengths $\left.\left(l_{\alpha_{1}}, l_{\alpha_{2}}, 0\right)\right)$, the segments $H_{11}$ and $H_{11}^{\prime}$ also match, so that the union of both segments is the geodesic $\delta_{1}$. Therefore, $\delta_{1}$ intersects $\alpha_{1}$ orthogonally, and so the twist parameter $t_{\alpha_{1}}$ is zero. In the same way, $\delta_{2}$ intersects $\alpha_{2}$ orthogonally and $t_{\alpha_{2}}=0$.

It is not difficult to find the expression for the length of $\beta$ in the FenchelNielsen coordinates $\left(l_{\alpha_{1}}, l_{\alpha_{2}}, t_{\alpha_{1}}, t_{\alpha_{2}}\right)$, either using trigonometry or by looking at the trace of the element representing $\beta$ in the corresponding fuchsian group. This is done in detail in [3]. We have

$$
\cosh \frac{l_{\beta}}{2}=\frac{1+\cosh \frac{l_{\alpha_{1}}}{2} \cosh \frac{l_{\alpha_{2}}}{2}}{\sinh \frac{l_{\alpha_{1}}}{2} \sinh \frac{l_{\alpha_{2}}}{2}} \cosh \frac{t_{\alpha_{1}}}{2} \cosh \frac{t_{\alpha_{2}}}{2}+\sinh \frac{t_{\alpha_{1}}}{2} \sinh \frac{t_{\alpha_{2}}}{2} .
$$

Computing the derivatives $\partial l_{\beta} / \partial l_{\alpha_{i}}$ directly from this formula we get

$$
\begin{aligned}
& -\sinh \frac{l_{\beta}}{2} \frac{\partial l_{\beta}}{\partial l_{\alpha_{1}}}=\frac{\cosh \frac{l_{\alpha_{1}}}{2}+\cosh \frac{l_{\alpha_{2}}}{2}}{\sinh ^{2} \frac{l_{\alpha_{1}}}{2} \sinh \frac{l_{\alpha_{2}}}{2}} \cosh \frac{t_{\alpha_{1}}}{2} \cosh \frac{t_{\alpha_{2}}}{2}, \\
& -\sinh \frac{l_{\beta}}{2} \frac{\partial l_{\beta}}{\partial l_{\alpha_{2}}}=\frac{\cosh \frac{l_{\alpha_{1}}}{2}+\cosh \frac{l_{\alpha_{2}}}{2}}{\sinh \frac{l_{\alpha_{1}}}{2} \sinh ^{2} \frac{l_{\alpha_{2}}}{2}} \cosh \frac{t_{\alpha_{1}}}{2} \cosh \frac{t_{\alpha_{2}}}{2} .
\end{aligned}
$$

Therefore, the equations determining the line of minima are:

$$
t_{\alpha_{1}}=t_{\alpha_{2}}=0, \quad \frac{a_{1}}{a_{2}}=\frac{\sinh \left(l_{\alpha_{2}} / 2\right)}{\sinh \left(l_{\alpha_{1}} / 2\right)} .
$$

By allowing $a_{1}, a_{2}$ to vary among all positive numbers, we observe that the corresponding lines of minima are pairwise disjoint and in fact foliate the whole plane $\left\{\left(l_{\alpha_{1}}, l_{\alpha_{2}}, t_{\alpha_{1}}, t_{\alpha_{2}}\right) \mid t_{\alpha_{1}}=0, t_{\alpha_{2}}=0\right\}$ in $\operatorname{Teich}\left(S_{1,2}\right)$.

Clearly, at one end of $\mathcal{L}_{\mu, \beta}$ the lengths $l_{\alpha_{1}}, l_{\alpha_{2}}$ tend to zero. This cannot happen when $s \rightarrow 1$ because if $l_{\alpha_{1}}, l_{\alpha_{2}} \rightarrow 0$, then $l_{\beta}$ and hence $(1-s) l_{\mu}\left(m_{s}\right)+$ $s l_{\beta}\left(m_{s}\right)$ tend to $\infty$, and this contradicts the fact that $m_{s}$ is the minimum. Thus $l_{\alpha_{1}}, l_{\alpha_{2}} \rightarrow 0$ as $s \rightarrow 0$ and therefore by Lemma $2.2(\mathrm{~b})$, the line $\mathcal{L}_{\mu, \beta}$ converges to a point of the form $\left[a_{1}^{\prime} \alpha_{1}+a_{2}^{\prime} \alpha_{2}\right]$, for some $a_{1}^{\prime}, a_{2}^{\prime} \geq 0$. To compute these 
numbers, we compute the lengths of the dual curves $\delta_{1}, \delta_{2}$. By hyperbolic trigonometry we get

$$
\cosh \frac{l_{\delta_{1}}}{4}=\sinh \frac{l_{\alpha_{2}}}{2} \sinh \frac{l_{\beta}}{2}, \quad \cosh \frac{l_{\delta_{2}}}{4}=\sinh \frac{l_{\alpha_{1}}}{2} \sinh \frac{l_{\beta}}{2} .
$$

Thus comparing the lengths of $\delta_{1}, \delta_{2}$ along the line of minima $\mathcal{L}_{\mu, \beta}$ we find

$$
\lim _{s \rightarrow 0} \frac{\cosh \left(l_{\delta_{1}} / 4\right)}{\cosh \left(l_{\delta_{2}} / 4\right)}=\lim _{s \rightarrow 0} \frac{\sinh \left(l_{\alpha_{2}} / 2\right)}{\sinh \left(l_{\alpha_{1}} / 2\right)}=\frac{a_{1}}{a_{2}} .
$$

Since $l_{\alpha_{i}} \rightarrow 0$, we have that $l_{\delta_{i}} \rightarrow \infty$, so that

$$
\frac{a_{1}}{a_{2}}=\lim _{s \rightarrow 0} e^{\left(l_{\delta_{1}}-l_{\delta_{2}}\right) / 2} .
$$

Taking logarithms, we get that $\lim _{s \rightarrow 0}\left(l_{\delta_{1}}-l_{\delta_{2}}\right)$ is a constant, and this implies that $\lim _{s \rightarrow 0}\left(l_{\delta_{1}} / l_{\delta_{2}}\right)=1$. Hence, $\mathcal{L}_{\mu, \beta} \rightarrow\left[\alpha_{1}+\alpha_{2}\right]$ as $s \rightarrow 0$.

\section{Statements of main results: pants decomposition case}

In order to prove Theorem 1.1, we need to show that the lengths of all the simple closed geodesics converge projectively to their intersection numbers with $\sum \alpha_{i}$. So we want to estimate the length of any simple closed geodesic along the line of minima. We first prove that along the line of minima the lengths of $\alpha_{i}$ tend to zero and the twist parameters about $\alpha_{i}$ are bounded (Proposition 4.1 (a) and (b)). When $\alpha_{i}$ is a pants decomposition, these two properties allow one to give a nice estimate of the length of a closed geodesic (Proposition 4.2): the main contribution is given by the arcs going through the collars around the curves $\alpha_{i}$. Finally, to compare the length of two closed geodesics, we need to compare the orders of the lengths $l_{\alpha_{i}}, l_{\alpha_{j}}$. This is done in Proposition 4.1 (c).

In this section we state these two propositions in the case in which $|\mu|$ is a pants decomposition and $\nu$ is rational. The propositions will be proved in the next section. Both propositions remain true when $\nu$ (in Proposition 4.1) and $\gamma$ (in Proposition 4.2) are arbitrary measured laminations. We will comment on the proof of these stronger versions in Section 5.4.

Recall that two real functions $f(s), g(s)$ have the same order as $s \rightarrow s_{0}$, denoted by $f \sim g$, when there exist positive constants $k<K$ so that $k<f(s) / g(s)<K$ for all $s$ near enough to $s_{0}$. Write $f(s)=O(1)$ if $f(s)$ is bounded. 
Proposition 4.1 Suppose that $\mu=\sum a_{i} \alpha_{i}$ and $\nu=\sum b_{i} \beta_{i}$ are two measured laminations where $\left\{\alpha_{i}\right\}$ is a pants decomposition, and $a_{i}>0$ for all $i$. Let $m_{s}$ be the minimum point of the function $F_{s}$. Then

(a) for any $i, \lim _{s \rightarrow 0} l_{\alpha_{i}}\left(m_{s}\right)=0$;

(b) for any $i,\left|t_{\alpha_{i}}\left(m_{s}\right)\right|$ is bounded when $s \rightarrow 0$;

(c) for all $i, j, l_{\alpha_{i}}\left(m_{s}\right) \sim l_{\alpha_{j}}\left(m_{s}\right) \sim s$ as $s \rightarrow 0$.

The proof of Proposition 4.1 (a) is direct and could be read now.

Proposition 4.2 Let $\left\{\alpha_{1}, \ldots, \alpha_{N}\right\}$ be a pants decomposition of $S$ and $\gamma$ a closed geodesic. Let $\rho_{n} \in \operatorname{Teich}(S)$ be a sequence so that, when $n \rightarrow \infty$, all the lengths $l_{\alpha_{i}}\left(\rho_{n}\right)$ are bounded above and the twists $t_{\alpha_{i}}\left(\rho_{n}\right)$ are bounded for all $i$. Then, as $n \rightarrow \infty$, we have

$$
l_{\gamma}\left(\rho_{n}\right)=2 \sum_{j=1}^{N} i\left(\alpha_{j}, \gamma\right) \log \frac{1}{l_{\alpha_{j}}\left(\rho_{n}\right)}+O(1) .
$$

In view of this proposition, it is enough to work with collars around $\alpha_{j}$ of width $2 \log \left(1 / l_{\alpha_{j}}\right)$, even if they are not the maximal embedded collars. The more relaxed hypothesis about the lengths $l_{\alpha_{i}}$ being bounded above is not needed if $\left\{\alpha_{1}, \ldots, \alpha_{N}\right\}$ is a pants decomposition, but will be useful in the general case in Section 6 .

\section{Proof of Theorem 1.1 for the pants decomposition case}

Suppose $\alpha_{1}, \ldots, \alpha_{N}$ is a pants decomposition system. By Proposition 4.1 (a), the lengths $l_{\alpha_{i}}$ tend to zero as $s \rightarrow 0$. Therefore, by Lemma $2.2(\mathrm{~b}), m_{s} \rightarrow$ $\left[a_{1}^{\prime} \alpha_{1}+\cdots+a_{N}^{\prime} \alpha_{N}\right]$, as $s \rightarrow 0$, for some $a_{i}^{\prime} \geq 0$. By Proposition 4.1 (b), along the line of minima the twists $t_{\alpha_{i}}$ are bounded. Then we can use Proposition 4.2 to estimate the length of two simple closed curves $\gamma, \gamma^{\prime}$ : the proportion between their lengths is

$$
\frac{l_{\gamma}}{l_{\gamma^{\prime}}}=\frac{2 \sum i\left(\alpha_{j}, \gamma\right) \log \frac{1}{l_{\alpha_{j}}}+O(1)}{2 \sum i\left(\alpha_{j}, \gamma^{\prime}\right) \log \frac{1}{l_{\alpha_{j}}}+O(1)} .
$$

Now, by Proposition 4.1(c), $l_{\alpha_{i}} \sim l_{\alpha_{j}}$ as $s \rightarrow 0$; this implies that $\log \frac{1}{l_{\alpha_{i}}} / \log \frac{1}{l_{\alpha_{j}}}$ $\rightarrow 1$ as $s \rightarrow 0$ (see Lemma 5.3 below). Dividing numerator and denominator of (3) by $\log \left(1 / l_{\alpha_{1}}\right)$, we get that $\lim _{s \rightarrow 0}\left(l_{\gamma} / l_{\gamma^{\prime}}\right)=i\left(\sum \alpha_{j}, \gamma\right) / i\left(\sum \alpha_{j}, \gamma^{\prime}\right)$. Hence $a_{i}^{\prime}=1$ for all $i$. 


\section{Proof: pants decomposition case}

We shall estimate the length of a geodesic $\gamma$ comparing it with the length of a "broken arc" relative to a pants decomposition. Broken arcs are a main tool in [13], and we refer there for details. The idea is that there is a unique curve freely homotopic to $\gamma$ with no backtracking, made up of arcs which wrap around a pants curve, alternating with arcs which cross a pair of pants from one boundary to another following the common perpendiculars between the boundaries. This collection of mutually perpendicular arcs constitute the broken arc, whose length, as shown in Lemma 5.1, approximates the length of $\gamma$.

To determine the length of the broken arc, we study the geometry of a pair of pants. By using the trigonometric formulae for right angle hexagons and pentagons, we can compute the length of the segments perpendicular to two boundary components, and estimate this length when the lengths of the boundary components tend to zero. This is done in Lemma 5.4.

\subsection{Broken arcs}

A broken arc in $\mathbb{H}^{2}$ is a sequence of oriented segments such that the final point of one segment is equal to the initial point of the next, and such that consecutive arcs meet orthogonally. Labelling the segments in order $V_{1}, H_{1}, \ldots, V_{r}, H_{r}, V_{r+1}$, we also require that for $1 \leq i \leq r-1$ the segments $H_{i}, H_{i+1}$ are contained in opposite halfplanes with respect to $V_{i+1}$. We call the $V_{i}$ the 'vertical arcs' and the $H_{i}$ the 'horizontal' ones.

Lemma 5.1 Consider a broken arc in hyperbolic plane with endpoints $R, R^{\prime}$ and with side lengths $s_{1}, d_{1}, \ldots, s_{r}, d_{r}, s_{r+1}$. For any $D>0$, there exists a constant $K=K(D, r)$, depending only on $D$ and the number $r$ of horizontal arcs, so that, if $d_{j}>D$ for all $j$, we have

$$
d\left(R, R^{\prime}\right)>\sum d_{j}+\sum s_{j}-K .
$$

If $D^{\prime}>D$, then $K\left(D^{\prime}, r\right)<K(D, r)$.

In the proof we use the following facts about universal constants for hyperbolic triangles, which can be deduced from the property that hyperbolic triangles are thin, see for example [5]. (I) There exists a positive constant $K\left(\theta_{0}\right)$ so that for any hyperbolic triangle with side lengths $a, b, c$ and angle $\theta$ opposite to $c$ satisfying $\theta \geq \theta_{0}>0$, we have $c>a+b-K\left(\theta_{0}\right)$. Moreover, if $\theta_{0}^{\prime}>\theta_{0}$, then 
$K\left(\theta_{0}^{\prime}\right)<K\left(\theta_{0}\right)$. (II) Given $D>0$, there exists a constant $\theta_{0}=\theta_{0}(D)$ so that for any hyperbolic triangle with one side of length $d \geq D$ and angles $\pi / 2, \theta$ on this side, we have $\theta \leq \theta_{0}$. If $D^{\prime}>D$, then $\theta_{0}\left(D^{\prime}\right)<\theta_{0}(D)$.

Proof of Lemma 5.1 Consider $D>0$. The proof will be by induction on $r$. For $r=1$ we have a broken arc with three arcs $V_{1}, H_{1}, V_{2}$ with lengths $s_{1}, d_{1}, s_{2}$; denote by $Q, Q^{\prime}$ the vertices of the arc $H_{1}$. Since $d_{1}>D$, by (II), there exists $\theta_{0}$ so that the angle $Q Q^{\prime} R$ is less than $\theta_{0}$; therefore the angle $R Q^{\prime} R^{\prime}$ is greater than $\theta_{1}=\pi / 2-\theta_{0}$. Applying (I) to the triangles $R Q Q^{\prime}$ and $R Q^{\prime} R^{\prime}$, we have

$$
d\left(R, R^{\prime}\right)>d\left(R, Q^{\prime}\right)+d\left(Q^{\prime}, R^{\prime}\right)-K\left(\theta_{1}\right)>s_{1}+d_{1}+s_{2}-K(\pi / 2)-K\left(\theta_{1}\right)
$$

so that we can take $K(D, 1)=K(\pi / 2)+K\left(\theta_{1}\right)$.

Now consider a broken arc with arc lengths $s_{1}, d_{1}, \ldots, s_{r}, d_{r}, s_{r+1}$ and $d_{i}>D$. Denote by $Q, Q^{\prime}$ the vertices of the arc $d_{r}$. Since $d_{r} \geq D$, the angle $R^{\prime} Q Q^{\prime}$ is smaller than $\theta_{0}$. Since $R, R^{\prime}$ are on different sides of the line containing the vertical segment $V_{r}$, the angle $R Q R^{\prime}$ is greater than $\theta_{1}=\pi / 2-\theta_{0}$. Applying (I) to the triangles $R Q R^{\prime}$ and $Q Q^{\prime} R^{\prime}$ and using the induction hypothesis we get

$$
d\left(R, R^{\prime}\right)>\sum_{j=1}^{r} d_{j}+\sum_{j=1}^{r+1} s_{j}-K(D, r-1)-K(\pi / 2)-K\left(\theta_{1}\right) .
$$

So we can take $K(D, r)=r\left(K\left(\frac{\pi}{2}\right)+K\left(\theta_{1}\right)\right)$. If $D^{\prime}>D$, by (I) and (II), we have that $K\left(D^{\prime}, r\right)<K(D, r)$.

Now let $\gamma$ be a closed geodesic on a hyperbolic surface $\rho$, and let the geodesics $\left\{\alpha_{i}\right\}$ be a pants decomposition. We shall use the $\left\{\alpha_{i}\right\}$ to construct a broken arc $B A_{\gamma}(\rho)$ associated to $\gamma$, as illustrated in Figure 2. Fix an orientation on $\gamma$ and let $Q$ be an intersection point of $\gamma$ with a pants curve. Let $\tilde{\gamma}$ be the lift of $\gamma$ through a lift $\tilde{Q}$ of $Q$. Let $\tilde{C}_{1}, \ldots, \tilde{C}_{r+1}$ be the lifts of the geodesics $\left\{\alpha_{i}\right\}$ which are intersected, in order, by $\tilde{\gamma}$, so that $\tilde{C}_{1} \cap \tilde{\gamma}=\tilde{Q}$ and $\tilde{C}_{r+1}$ is the image of $\tilde{C}_{1}$ under the covering translation $\tau$ corresponding to $\gamma$. Thus, if we denote by $\tilde{Q}^{\prime}$ the intersection of $\tilde{\gamma}$ with $\tilde{C}_{r+1}$, the geodesic segment $\tilde{Q} \tilde{Q}^{\prime}$ projects onto $\gamma$. For $i=1, \ldots, r$, consider the common perpendicular segment to $\tilde{C}_{i}, \tilde{C}_{i+1}$, with endpoints denoted by $Q_{i}^{-}, Q_{i}^{+}$; and finally, let $Q_{0}^{+}=\tau^{-1}\left(Q_{r}^{+}\right)$. Then we define $B A_{\gamma}(\rho)$ to be the broken arc with vertical arcs the segments $Q_{0}^{+} Q_{1}^{-}, Q_{1}^{+} Q_{2}^{-}, \ldots, Q_{r-1}^{+} Q_{r}^{-}$, and horizontal arcs the segments $Q_{1}^{-} Q_{1}^{+}, \ldots, Q_{r}^{-} Q_{r}^{+}$. Denote by $s_{i}$ the lengths of the vertical arcs and by $d_{i}$ the lengths of the horizontal arcs. The horizontal segments project onto 


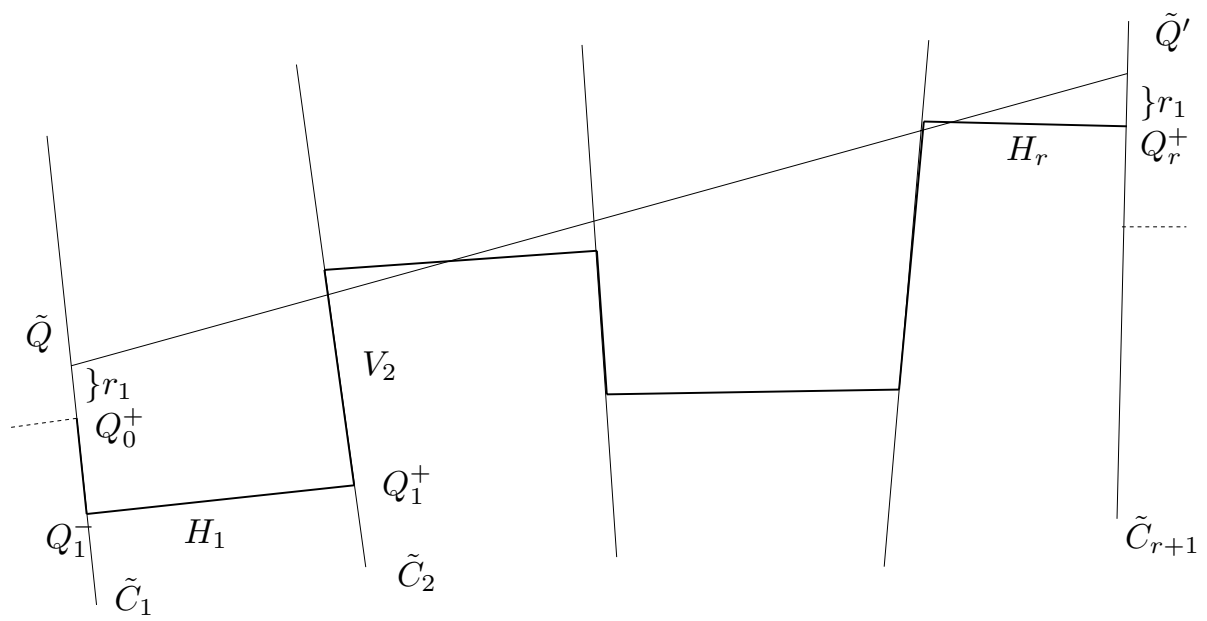

Figure 2: Broken arc with $r=4$

geodesic segments which are the perpendiculars either between two boundary components, or from one boundary component to itself, of one of the pairs of pants. Their length will be studied in the next subsection. The vertical segments project onto arcs contained in the pants geodesics $\alpha_{j}$. If the segment $Q_{i}^{+} Q_{i+1}^{-}$projects, say, onto $\alpha_{1}$, then its length is of the form

$$
s_{i}=\left|n_{i} l_{\alpha_{1}}+t_{\alpha_{1}}+e_{i}\right|
$$

where $n_{i} \in \mathbb{Z}$ depends on the combinatorics of $\gamma$ relative to the pants decomposition (related to how many times $\gamma$ wraps around $\alpha_{1}$ ), and $e_{i}$ is a number smaller in absolute value than $l_{\alpha_{1}}$ which depends on the combinatorics of $\gamma$ and on the geometry of the two pairs of pants meeting along $\alpha_{1}$. For our purposes we will not need more details about $e_{i}$, see [13] for more explanation.

We remark that the endpoints of $B A_{\gamma}(\rho)$ do not necessarily coincide with those of $\tilde{\gamma}$, but we can consider another broken arc $\overline{B A}_{\gamma}$ with the same endpoints as $\tilde{\gamma}$ by just changing the first vertical segment $Q_{0}^{+} Q_{1}^{-}$to $\tilde{Q} Q_{1}^{-}$and adding at the end the vertical segment $Q_{r}^{+} \tilde{Q}^{\prime}$. To control the lengths of these two new segments, we use the following lemma.

Lemma 5.2 With the above notation, suppose that $\tilde{C}_{i}$ projects onto $\alpha_{k}$ and denote $\tilde{Q}_{i}=\tilde{\gamma} \cap \tilde{C}_{i}$. Then, either $\tilde{Q}_{i}$ is between $Q_{i-1}^{+}$and $Q_{i}^{-}$, or the minimum of the distances $d\left(Q_{i-1}^{+}, \tilde{Q}_{i}\right), d\left(Q_{i}^{-}, \tilde{Q}_{i}\right)$ is less than $l_{\alpha_{k}}$.

Proof Suppose that $\tilde{Q}_{i}$ is not between $Q_{i-1}^{+}$and $Q_{i}^{-}$and that both distances $d\left(Q_{i-1}^{+}, \tilde{Q}_{i}\right), d\left(Q_{i}^{-}, \tilde{Q}_{i}\right)$ are greater than $l_{\alpha_{k}}$. Then, applying the covering trans- 
formation corresponding to $\alpha_{k}$ (or to $\alpha_{k}^{-1}$ ) to the segments $H_{i-1}, H_{i}$, we obtain two new segments $H_{i-1}^{\prime}, H_{i}^{\prime}$ which are closer to $\tilde{\gamma}$ than $H_{i-1}, H_{i}$. The lines containing these segments are disjoint from $\tilde{C}_{i-1}, \tilde{C}_{i+1}$ respectively and hence necessarily both intersect $\tilde{\gamma}$. Therefore they determine, together with the lines $\tilde{\gamma}, \tilde{C}_{i}$, two right-angled triangles. One of them has angle sum greater than $\pi$, so we have a contradiction. (There is a similar argument when one of the distances $d\left(Q_{i-1}^{+}, \tilde{Q}_{i}\right), d\left(Q_{i}^{-}, \tilde{Q}_{i}\right)$ is equal to $\left.l_{\alpha_{k}}.\right)$

As a consequence, if $\tilde{C}_{1}$ projects over $\alpha_{i_{1}}$ and if $\bar{s}_{1}, \bar{s}_{r+1}$ are the lengths of the first and last vertical segments of $\overline{B A}_{\gamma}$, then $\bar{s}_{1}+\bar{s}_{r+1}$ is either equal to $s_{1}$ or to $s_{1}+2 r_{1}$, where $r_{1}<l_{\alpha_{i_{1}}}$. Thus, by Lemmas 5.1 and 5.2 , we can approximate the length of $\gamma$ by the length of the broken $\operatorname{arc} B A_{\gamma}$ within an error of $K(D, r)+2 l_{\alpha_{i_{1}}}$.

Remark A straightforward generalisation of the above construction allows one to associate a broken arc with any (not necessarily closed) geodesic, and also with a geodesic arc with endpoints on the pants curves. (For a geodesic arc, to determine the first vertical arc, prolong the geodesic in the negative direction until it crosses the next pants curve.) Then, we can use Lemmas 5.1 and 5.2 to estimate this length from the length of this broken arc. This is useful when $\nu$ is irrational, see Section 5.4.

\subsection{Geometry of a pair of pants}

We now estimate the lengths of the common perpendicular segments between two curves of the pants decomposition. In the situation to be considered, these segments will be sufficiently long to apply Lemma 5.1.

It is useful to refine slightly the notation $f(s) \sim g(s)$ as $s \rightarrow s_{0}$ defined on p. 216. For $f, g$ real valued functions we write $f \approx g$ to mean that $\lim _{s \rightarrow s_{0}} f / g$ exists and is strictly positive. Clearly, $f \approx g$ is slightly stronger than $f \sim g$. However even if the limit does not exist, if $f \sim g$, and if both functions tend to either 0 or $\infty$, then $\lim _{s \rightarrow s_{0}} \log f / \log g$ does exist and equals 1 . This fact is crucial for our results. We collect this and other elementary properties in the next lemma. We also recall the notation $f=O(g)$ as $s \rightarrow s_{0}$ meaning that $f / g$ is bounded when $s \rightarrow s_{0}$, and $f=o(g)$ as $s \rightarrow s_{0}$ meaning that $f / g \rightarrow 0$ when $s \rightarrow s_{0}$.

Lemma $5.3 \quad$ (a) $f \sim g$ is equivalent to $\log f=\log g+O(1)$. 
(b) If $f, g \rightarrow \infty$ or 0 and $f \sim g$, then $\lim (\log f / \log g)=1$.

(c) $f \approx g$ is equivalent to $f=a g+o(g)$, with $a>0$.

Proof (a) If there exists $0<k<K$ with $k<f / g<K$, then taking $\operatorname{logarithms}$ we get that $\log k<\log f-\log g<\log K$. The converse is also clear by exponentiating $\log f=\log g+O(1)$.

(b) Since $g \rightarrow 0$ or $\infty$, then $\log g \rightarrow-\infty$ or $+\infty$ respectively, and in both cases $O(1) / \log g \rightarrow 0$. Then, dividing $\log f=\log g+O(1)$ by $\log g$, we get the result. Part (c) is immediate from the definitions.

Lemma 5.4 Consider a pair of pants $P$ with boundary components $B_{1}, B_{2}$, $B_{3}$ of lengths $l_{1}, l_{2}, l_{3}$. For any $i, j \in\{1,2,3\}$, let $H_{i j}$ be the common perpendicular arc to the boundary components $B_{i}, B_{j}$, with length $d_{i j}$. Suppose that each of $l_{1}, l_{2}, l_{3}$ either tends to zero or is bounded above. Then, for any $i, j$, we have

$$
d_{i j}=\log \frac{1}{l_{i}}+\log \frac{1}{l_{j}}+O(1) .
$$

Proof The pair of pants $P$ is made up by gluing two isometric right angle hexagons with alternate sides of lengths $l_{1} / 2, l_{2} / 2, l_{3} / 2$. For $i \neq j$, the segments $H_{i j}$ are the remaining sides. The segment $H_{i i}$ is the union of the common perpendicular segments in the two hexagons between the side contained in $B_{i}$ and its opposite side. We therefore obtain the trigonometric formulae:

$$
\cosh d_{i j}=\frac{\cosh \frac{l_{k}}{2}+\cosh \frac{l_{i}}{2} \cosh \frac{l_{j}}{2}}{\sinh \frac{l_{i}}{2} \sinh \frac{l_{j}}{2}}, \quad \cosh \frac{d_{i i}}{2}=\sinh d_{i j} \sinh \frac{l_{j}}{2} .
$$

For $i \neq j$ we deduce that $\cosh d_{i j} \approx \frac{1}{l_{i} l_{j}}$ as $\left(l_{1}, l_{2}, l_{3}\right) \rightarrow(0,0,0)$. Thus there exits $a>0$ so that

$$
\cosh d_{i j}=\frac{a}{l_{i} l_{j}}+o\left(\frac{1}{l_{i} l_{j}}\right) \text {. }
$$

Since $d_{i j} \rightarrow \infty, e^{-d_{i j}}$ is bounded, and so

$$
e^{d_{i j}}=\frac{2 a}{l_{i} l_{j}}+o\left(\frac{1}{l_{i} l_{j}}\right) .
$$

The result follows from Lemma 5.3 (a).

For the case $i=j$, we have that $\sinh d_{i j} \approx \frac{1}{l_{i} l_{j}}$ as $\left(l_{1}, l_{2}, l_{3}\right) \rightarrow(0,0,0)$ (because $d_{i j} \rightarrow \infty$ and in that case $\sinh d_{i j} \approx \cosh d_{i j}$ ). Then, from the above formula for $\cosh \left(d_{i i} / 2\right)$, we have that $\cosh \left(d_{i i} / 2\right) \approx 1 / l_{i}$. As before, $d_{i i} / 2=\log \left(1 / l_{i}\right)+O(1)$ and so we get the result.

We can check that the same works when some or all the $l_{i}$ do not tend to zero but are still bounded above. 


\subsection{Proof of Propositions 4.1 and 4.2}

Proposition 4.2 is used in the proof of Proposition 4.1 (c), so we follow this order below. We remark that in our proofs the hypothesis of being in the line of minima is always used in the same way: simply compare the value of $F_{s}$ at its minimum $m_{s}$ and at some other point.

Proof of Proposition 4.1(a) Consider $i$ with $a_{i} \neq 0$. Given $\epsilon>0$, consider a hyperbolic surface $\rho_{\epsilon}$ so that the length of any $\alpha_{i}$ is equal to $\left(a_{i} / 4\right) \epsilon$. Take $s_{0}=\min \left\{\frac{1}{2}, \frac{a_{i} \epsilon}{4 l_{\nu}\left(\rho_{\epsilon}\right)}\right\}$. Then, for $s<s_{0}$, we have

$$
\begin{aligned}
F_{s}\left(\rho_{\epsilon}\right) & =(1-s) \sum_{j=1}^{N} a_{j} l_{\alpha_{j}}\left(\rho_{\epsilon}\right)+s l_{\nu}\left(\rho_{\epsilon}\right) \\
& =(1-s)\left(\sum_{j=1}^{N} a_{j}\right) \frac{a_{i}}{4} \epsilon+s l_{\nu}\left(\rho_{\epsilon}\right)<\frac{a_{i}}{4} \epsilon+\frac{a_{i}}{4} \epsilon=\frac{a_{i}}{2} \epsilon .
\end{aligned}
$$

Hence, for the minimum point $m_{s}$ of $F_{s}$, we have

$$
(1-s) a_{i} l_{\alpha_{i}}\left(m_{s}\right) \leq F_{s}\left(m_{s}\right)<\left(a_{i} / 2\right) \epsilon .
$$

Since $1-s>1 / 2$, we have that $l_{\alpha_{i}}\left(m_{s}\right)<\epsilon$. Since, by hypothesis, $a_{i} \neq 0$ for all $i$, we have the result.

Proof of Proposition 4.1(b) We shall prove that all the twists about $\alpha_{r}$ are bounded when $s \rightarrow 0$. Suppose not; renumbering, we may assume that $\left|t_{\alpha_{1}}\left(m_{s}\right)\right|$ is not bounded. Suppose moreover that, up to subsequence, $t_{\alpha_{1}} \rightarrow$ $+\infty$. (The proof is the same if $t_{\alpha_{1}} \rightarrow-\infty$.)

For each $s$, consider the point $\rho_{s}$ obtained from $m_{s}$ by twisting (earthquaking) by $-t_{\alpha_{1}}\left(m_{s}\right)$ about $\alpha_{1}$. This new surface has the same Fenchel-Nielsen coordinates with respect to $\left\{\alpha_{i}\right\}$ as $m_{s}$ (for a fixed choice of dual curves) except that $t_{\alpha_{1}}\left(\rho_{s}\right)=0$. We shall prove that, if $s$ is small enough, then $l_{\nu}\left(m_{s}\right)>l_{\nu}\left(\rho_{s}\right)$. Since the lengths of $\alpha_{i}$ are the same at both points, we will have that $F_{s}\left(m_{s}\right)>F_{s}\left(\rho_{s}\right)$, which is a contradiction.

We make the following argument for all the curves $\beta$ in the support of $\nu$. Since the lengths of all the pants curves tend to zero, by Lemma 5.4 there exists $s_{0}>0$ so that for all $s<s_{0}$, all the horizontal arc lengths $d_{i j}$ of the broken arc $B A_{\beta}$ are bounded below by some constant $D$, and therefore by Lemma 5.1 (and Lemma 5.2), there exists a constant $K$ so that $l_{B A_{\beta}}\left(m_{s}\right)-l_{\beta}\left(m_{s}\right)<K$ for all $s<s_{0}$. We will prove that

$$
l_{B A_{\beta}}\left(m_{s}\right)-l_{B A_{\beta}}\left(\rho_{s}\right) \rightarrow \infty \text { as } s \rightarrow 0 .
$$

Assuming (5), there exists $0<s_{1}<s_{0}$ so that for all $s<s_{1}, l_{B A_{\beta}}\left(m_{s}\right)-$ $l_{B A_{\beta}}\left(\rho_{s}\right)>K$, and then

$$
l_{\beta}\left(m_{s}\right)>l_{B A_{\beta}}\left(m_{s}\right)-K>l_{B A_{\beta}}\left(\rho_{s}\right)>l_{\beta}\left(\rho_{s}\right) .
$$


Summing over all curves $\beta_{i}$, we obtain $l_{\nu}\left(m_{s}\right)>l_{\nu}\left(\rho_{s}\right)$.

It is left to prove (5). We compare the broken $\operatorname{arcs} B A_{\beta}$ at the points $m_{s}$ and $\rho_{s}$. The horizontal arcs have the same length in both broken arcs. In fact only the vertical arcs projecting over the curve $\alpha_{1}$ change length. There are $i\left(\beta, \alpha_{1}\right)$ of such segments, with lengths $s_{1}, \ldots, s_{i\left(\beta, \alpha_{1}\right)}$ at $m_{s}$, where $s_{j}=\left|n_{j} l_{\alpha_{1}}+t_{\alpha_{1}}+e_{j}\right|$. On the other hand, the lengths of these segments at $\rho_{s}$ are $s_{j}^{\prime}=\left|n_{j} l_{\alpha_{1}}+e_{j}\right|$. (We remark that $n_{1}+\cdots+n_{i\left(\beta, \alpha_{1}\right)}$ is called the wrapping number of $\beta$ around $\alpha_{1}$.) Then

$$
l_{B A_{\beta}}\left(m_{s}\right)-l_{B A_{\beta}}\left(\rho_{s}\right)=\sum\left(s_{j}-s_{j}^{\prime}\right) \geq i\left(\beta, \alpha_{1}\right) t_{\alpha_{1}}-2 \sum_{j=1}^{i\left(\beta, \alpha_{1}\right)}\left|n_{j} l_{\alpha_{1}}+e_{j}\right| .
$$

Since $\left|n_{j} l_{\alpha_{1}}+e_{j}\right|$ is bounded for all $j$, this expression tends to infinity as $s \rightarrow 0$ as required.

Proof of Proposition 4.2 Consider the broken arc $B A_{\gamma}\left(\rho_{n}\right)$ associated to $\gamma$. Since all the lengths $l_{\alpha_{i}}$ tend to 0 or are bounded above when $n \rightarrow \infty$, then, for $n$ big enough, all horizontal arcs of this broken arc are greater than some given $D$. Then we can use Lemmas 5.1 and 5.2 to estimate the length of $\gamma$ and we have $\sum d_{i}+\sum s_{i}-K<l_{\gamma}\left(\rho_{n}\right)<\sum d_{i}+\sum s_{i}$. Since all the twists $t_{\alpha_{i}}$ are bounded, the lengths of the vertical arcs are bounded. On the other hand, using Lemma 5.4 to estimate the lengths $d_{i}$ of the horizontal arcs, and collecting terms together, we have

$$
l_{\gamma}\left(\rho_{n}\right)=\sum d_{i}+O(1)=\sum_{j} r_{j} \log \frac{1}{l_{\alpha_{j}}\left(\rho_{n}\right)}+O(1),
$$

where $r_{j}$ is the number of times that the projections of the horizontal arcs end in the geodesic $\alpha_{j}$. Then this number is equal to $2 i\left(\alpha_{j}, \gamma\right)$, and we get the desired result.

Proof of Proposition 4.1(c) We need to prove that, on the line of minima $\mathcal{L}_{\mu, \nu}$, all the lengths $l_{\alpha_{i}}$ have the same order as $s$, when $s \rightarrow 0$. That is, there exists $\epsilon>0$ and positive constants $k_{i}<K_{i}$ for any $i$, so that

$$
k_{i}<\frac{l_{\alpha_{i}}\left(m_{s}\right)}{s}<K_{i}
$$

for all $s<\epsilon$. Suppose not; then, up to subsequence, there exists some curve $\alpha_{j}$ so that, when $s \rightarrow 0$, then $l_{\alpha_{j}}\left(m_{s}\right) / s$ tends either to 0 or to $\infty$.

We construct a new sequence of surfaces $\rho_{s}$ defined by the Fenchel-Nielsen coordinates $l_{\alpha_{i}}=s$ and $t_{\alpha_{i}}=0$, for all $i$. We compare $F_{s}\left(m_{s}\right)$ with $F_{s}\left(\rho_{s}\right)$, 
and show that $F_{s}\left(m_{s}\right)-F_{s}\left(\rho_{s}\right)>0$, which will be a contradiction because $m_{s}$ is the minimum of the function $F_{s}$.

By Proposition 4.1 (a) and (b), along $m_{s}$ the lengths $l_{\alpha_{i}}$ tend to 0 and the twists $t_{\alpha_{i}}$ are bounded. Applying Proposition 4.2 to estimate the length of the curves $\beta_{i}$ we find

$$
F_{s}\left(m_{s}\right)=(1-s) \sum_{i} a_{i} l_{\alpha_{i}}\left(m_{s}\right)+s \sum_{i} b_{i}\left(2 \sum_{j} i\left(\alpha_{j}, \beta_{i}\right) \log \frac{1}{l_{\alpha_{j}}\left(m_{s}\right)}+O(1)\right) .
$$

On the other hand, the sequence $\rho_{s}$ also satisfies the hypothesis of Proposition 4.2 , and so we can also use this proposition to estimate the length of $\beta_{i}$, giving

$$
F_{s}\left(\rho_{s}\right)=(1-s) \sum_{i} a_{i} s+s \sum_{i} b_{i}\left(2 \sum_{j} i\left(\alpha_{j}, \beta_{i}\right) \log \frac{1}{s}+O(1)\right) .
$$

Then

$$
\begin{aligned}
& \frac{F_{s}\left(m_{s}\right)-F_{s}\left(\rho_{s}\right)}{s} \\
& =(1-s) \sum_{i} a_{i} \frac{l_{\alpha_{i}}\left(m_{s}\right)-s}{s} \\
& \quad \quad+\sum_{i} b_{i}\left(2 \sum_{j} i\left(\alpha_{j}, \beta_{i}\right)\left(\log \frac{1}{l_{\alpha_{j}}\left(m_{s}\right)}-\log \frac{1}{s}\right)+O(1)\right) \\
& \quad(1-s) \sum_{i} a_{i} \frac{l_{\alpha_{i}}\left(m_{s}\right)}{s}+\sum_{i} b_{i}\left(2 \sum_{j} i\left(\alpha_{j}, \beta_{i}\right)\left(\log \frac{s}{l_{\alpha_{j}}\left(m_{s}\right)}\right)\right)+O(1) \\
& =\sum_{i}\left((1-s) a_{i} \frac{l_{\alpha_{i}}\left(m_{s}\right)}{s}+C_{i} \log \frac{s}{l_{\alpha_{i}}\left(m_{s}\right)}\right)+O(1),
\end{aligned}
$$

where in the last equality we have rearranged the second group of summands, and $C_{i}$ are some positive coefficients.

Now, if there is some $i$ so that $l_{\alpha_{i}}\left(m_{s}\right) / s \rightarrow 0$, then $\log \left(s / l_{\alpha_{i}}\left(m_{s}\right)\right) \rightarrow \infty$. On the other hand, if there is some $i$ so that $l_{\alpha_{i}}\left(m_{s}\right) / s \rightarrow \infty$ then $\log \left(s / l_{\alpha_{i}}\left(m_{s}\right)\right) \rightarrow$ $-\infty$, but any positive linear combination of $l_{\alpha_{i}}\left(m_{s}\right) / s$ and $\log \left(s / l_{\alpha_{i}}\left(m_{s}\right)\right)$ tends to infinity.

Hence, $\left(F_{s}\left(m_{s}\right)-F_{s}\left(\rho_{s}\right)\right) / s$ tends to $+\infty$ and therefore $F_{s}\left(m_{s}\right)-F_{s}\left(\rho_{s}\right)>0$ for sufficiently small $s$, obtaining the desired contradiction.

\subsection{The proof of Theorem 1.1 when $\nu$ is irrational}

Finally we discuss the proof of Theorem 1.1 when $\nu$ is irrational. All that is needed is to extend Propositions 4.1 and 4.2 to the case in which $\nu$ and $\gamma$ respectively are general measured laminations. 
First, consider the effect of replacing $\gamma$ in Proposition 4.2 by an irrational lamination $\eta$. There exists a sequence of rational laminations $c_{k} \gamma_{k}$ converging to $\eta$, with $\gamma_{k}$ simple closed curves and $c_{k} \rightarrow 0$. Then $l_{\eta}\left(\rho_{n}\right)=\lim _{k \rightarrow \infty} c_{k} l_{\gamma_{k}}\left(\rho_{n}\right)$. We can compute this limit by using the expression obtained in Proposition 4.2 for closed curves. Since $\lim _{k \rightarrow \infty} i\left(c_{k} \gamma_{k}, \alpha\right)=i(\eta, \alpha)$, then we only need to check that the error (which is a function $f_{k}(n)$, bounded for fixed $k$ as $n \rightarrow \infty$ ) stays bounded when $k \rightarrow \infty$. By careful inspection of this error (in the proofs of Lemmas 5.1, 5.4 and Proposition 4.2), we see that it depends linearly on the intersection number of $\gamma_{k}$ with $\sum \alpha_{i}$ and on the wrapping numbers of $\gamma_{k}$ around $\alpha_{i}$. In both cases, these numbers, after scaling with $c_{k}$, converge when $k \rightarrow \infty$ (to the intersection number of $\eta$ with $\sum \alpha_{i}$ and to the twisting numbers of $\eta$ around $\alpha_{i}$ respectively). This proves Proposition 4.2.

The proof of Proposition 4.1 (b) for $\nu$ irrational, uses the same kind of arguments. The proof of part (c) is unchanged, once we have the stronger version of Proposition 4.2.

Alternatively, we can compute the length of an irrational lamination $\eta$ from its definition (see [6]), as the integral over the surface of the product measure $d \eta \times d l$, where $d l$ is the length measure along the leaves of $\eta$. We can cover the surface with thin rectangles, so that the length of arcs of $\eta$ intersecting one rectangle are almost equal, and we approximate the length of one of these geodesic arcs by the length of a broken arc (notice that we need the remark after Lemma 5.2 to do this). In this way we can prove both Propositions 4.2 and 4.1 (b).

\section{Non-pants decomposition case}

We now investigate the modifications needed to the above work if $\alpha_{1}, \ldots, \alpha_{N}$ is not a pants decomposition. The problem is that we no longer have full control over the geometry of the complement in $S$ of the curve system $\alpha_{1}, \ldots, \alpha_{N}$. The hyperbolic structures on at least some components of the complement might themselves diverge, in other words, the estimate of Proposition 4.2 for the lengths of arbitrary closed geodesics may no longer hold. Without ruling out this possibility, we show that the divergences in question must be of a lower order than those caused by the shrinking of the curves $\alpha_{i}$. A precise statement is made in Corollary 6.6.

In more detail, we proceed as follows. With minimal changes we still can prove that $l_{\alpha_{i}} \rightarrow 0$, with the same order as $s$, and that the twists $t_{\alpha_{i}}$ are bounded 
(Proposition 6.1). This implies that, if there exists a limiting lamination $\eta$, then its support is either disjoint from or contains the curves $\alpha_{i}$. Next we prove that there is in fact no other lamination contained in the limiting lamination, and therefore $[\eta]=\left[a_{1}^{\prime} \alpha_{1}+\cdots+a_{N}^{\prime} \alpha_{N}\right]$, for some coefficients $a_{i}^{\prime} \geq 0$ (Proposition 6.2). To compute these coefficients we need to compare the lengths of two closed geodesics. Even though we no longer have Proposition 4.2, we can still extend the curves $\alpha_{i}$ to a pants decomposition and estimate the length of the dual curves, (Proposition 6.5). This is enough to compute the coefficients $a_{i}^{\prime}$ and prove Theorem 1.1. A posteriori we obtain an estimate for the length of closed geodesics along the line of minima in Corollary 6.6.

Proposition 6.1 Suppose that $\mu=\sum a_{i} \alpha_{i}, \nu=\sum b_{i} \beta_{i}$ are two measured laminations (where $\left\{\alpha_{1}, \ldots, \alpha_{N}\right\}$ is not necessarily a pants decomposition), and that $a_{i}>0$ for all $i$. Let $m_{s}$ be the minimum point of the function $F_{s}$. Then:

(a) for any $i, \lim _{s \rightarrow 0} l_{\alpha_{i}}\left(m_{s}\right)=0$;

(b) for any $i,\left|t_{\alpha_{i}}\left(m_{s}\right)\right|$ is bounded when $s \rightarrow 0$;

(c) for all $i, j, l_{\alpha_{i}}\left(m_{s}\right) \sim l_{\alpha_{j}}\left(m_{s}\right) \sim s$ as $s \rightarrow 0$.

Remark The twist parameter $t_{\alpha_{i}}$ is a real parameter which determines how the surface is glued along $\alpha_{i}$. It is determined up to the choice of an initial surface on which the twist is zero. Changing the initial surface results in an additive change to the twist parameter, so (b) above is independent of this choice.

Proof The proof of (a) is exactly the same as that of Proposition 4.1(a).

For (b), consider a broken arc associated to the curve $\beta_{i}$ relative to the curves $\alpha_{1}, \ldots, \alpha_{N}$. Even if these curves are not a pants decomposition, the definition of broken arc given in Section 5.1 makes sense. The horizontal segments now project onto arcs perpendicular to two of the $\alpha_{i}$. If the length of each $\alpha_{i}$ is sufficiently small, the horizontal segments are greater that some given positive constant $D$ and we can apply Lemmas 5.1 and 5.2 to approximate the length of $\beta_{i}$ with the length of the corresponding broken arc. The length of the vertical arc projecting over $\alpha_{i}$ is $\left|t_{\alpha_{i}}+r\right|$, where $r$ depends only on the curve $\beta_{i}$ and the system $\alpha_{1}, \ldots, \alpha_{N}$. (To see this, think about obtaining the given surface from an initial surface with $t_{\alpha_{i}}=0$ by twisting about $\alpha_{i}$.) We can therefore argue exactly as in the proof of Proposition 4.1 (b): consider points $\rho_{s}$ obtained from 
the points $m_{s}$ on the line of minima by twisting by $-t_{\alpha_{i}}\left(m_{s}\right)$ about each $\alpha_{i}$. As in that proposition, we obtain that $F_{s}\left(m_{s}\right)>F_{s}\left(\rho_{s}\right)$ for small enough $s$.

For (c) we follow the same argument as in the proof of Proposition 4.1 (c). Let $\gamma_{1}, \ldots, \gamma_{K}$ be simple closed curves so that $\alpha_{i}, \gamma_{j}$ are a pants decomposition. Consider the surfaces $\rho_{s}$ defined by $l_{\alpha_{i}}\left(\rho_{s}\right)=s, l_{\gamma_{i}}\left(\rho_{s}\right)=1, t_{\alpha_{i}}\left(\rho_{s}\right)=t_{\gamma_{i}}\left(\rho_{s}\right)=$ 0 . The family $\rho_{s}$ satisfies the hypothesis of Proposition 4.2, and so we can estimate the length of $\beta_{i}$ as

$$
l_{\beta_{i}}\left(\rho_{s}\right)=2 \sum_{j} i\left(\alpha_{j}, \beta_{i}\right) \log \frac{1}{s}+O(1) .
$$

Now each curve $\alpha_{i}$ is contained in an embedded annular collar of width at least $2 \log \left(1 / l_{\alpha_{i}}\right)$. Using the contribution of the these collars gives the following rough estimation for the length of $\beta_{i}$ at $m_{s}$ :

$$
l_{\beta_{i}}\left(m_{s}\right)=2 \sum_{j} i\left(\alpha_{j}, \beta_{i}\right) \log \frac{1}{l_{\alpha_{j}}\left(m_{s}\right)}+f(s),
$$

where $f(s)$ is a positive function that might tend to infinity. Now, as in the proof of Proposition 4.1 (c), we have

$$
\frac{F_{s}\left(m_{s}\right)-F_{s}\left(\rho_{s}\right)}{s}=\sum_{i}\left((1-s) a_{i} \frac{l_{\alpha_{i}}\left(m_{s}\right)}{s}+C_{i} \log \frac{s}{l_{\alpha_{i}}\left(m_{s}\right)}\right)+f(s)-O(1)
$$

where $f(s)-O(1)$ may be negative but is nevertheless bounded below. The conclusion follows as in that proposition since, if $l_{\alpha_{i}}$ does not have the same order as $s$, the above group of summands always tends to $+\infty$.

Proposition 6.2 Let $\mu, \nu$ and $m_{s}$ be as in Proposition 6.1 and suppose that $m_{s}$ converges to a projective measured lamination $[\eta]$. Then the support of $[\eta]$ is contained in the union of $\alpha_{1}, \ldots, \alpha_{N}$.

Proof If the conclusion is false, then using Proposition 6.1 (a) we must have $|\eta| \subset \alpha_{1} \cup \cdots \cup \alpha_{N} \cup|\delta|$, where $\delta$ is a measured lamination whose support is disjoint from the $\alpha_{i}$. Since $\mu$ and $\nu$ fill up the surface and $i(\mu, \delta)=0$, it follows that $i(\nu, \delta) \neq 0$, and therefore some curve $\beta \subset|\nu|$ intersects $|\delta|$. Let $\bar{\kappa}$ be a geodesic arc contained in $\beta$, intersecting $\delta$, running from some $\alpha_{i}$ to some $\alpha_{j}$ (where possibly $\alpha_{i}=\alpha_{j}$ ) and not intersecting any other $\alpha_{l}$. We take open collar neighbourhoods $A_{i}$ of the curves $\alpha_{i}$, of width $2 \log \left(1 / l_{\alpha_{i}}\right)$, and let $\kappa=\bar{\kappa}-\bar{\kappa} \cap\left(A_{i} \cup A_{j}\right)$, so that $\kappa$ is a geodesic segment with endpoints on the relevant components $\tilde{\alpha}_{i}, \tilde{\alpha}_{j}$ of the boundaries $\partial A_{i}$ and $\partial A_{j}$. Note that the boundary curves $\tilde{\alpha}_{i}, \tilde{\alpha}_{j}$ of the collars $A_{i}, A_{j}$ have length $O(1)$. 
We are going to prove that $l_{\kappa}$, the length of the geodesic segment $\kappa$, tends to infinity by comparing to the length of a simple closed curve or curves we call the double of $\kappa$ (or $\bar{\kappa}$ ). If $i \neq j$ (or if $i=j$ but $\kappa$ meets both boundary components of the collar $A_{i}$ ), then the double is the simple closed curve $\tilde{\beta}$ created by going around $\tilde{\alpha}_{i}$, then parallel to $\kappa$, around $\tilde{\alpha}_{j}$, and back parallel to $\kappa$. In the case that $\alpha_{i}=\alpha_{j}$ and $\kappa$ intersects only one boundary component of the collar $A_{i}$, then $\kappa$ splits this boundary component, $\tilde{\alpha}_{i}$, into two arcs $\tilde{\alpha}_{i}^{\prime}$ and $\tilde{\alpha}_{i}^{\prime \prime}$; we create two simple closed curves $\tilde{\beta}^{\prime}=\kappa \cup \tilde{\alpha}_{i}^{\prime}$ and $\tilde{\beta}^{\prime \prime}=\kappa \cup \tilde{\alpha}_{i}^{\prime \prime}$, and designate $\tilde{\beta}=\tilde{\beta}^{\prime} \cup \tilde{\beta}^{\prime \prime}$ the double. In Lemma 6.3 below, we show that $i(\tilde{\beta}, \delta) \neq 0$, where in the second case we define $i(\tilde{\beta}, \delta)=i\left(\tilde{\beta}^{\prime}, \delta\right)+i\left(\tilde{\beta}^{\prime \prime}, \delta\right)$.

Since $m_{s} \rightarrow\left[\sum a_{i}^{\prime} \alpha_{i}+\delta\right]$, the length on the surface $m_{s}$ of the geodesic(s) isotopic to $\tilde{\beta}$ must tend to infinity as $s \rightarrow 0$. We claim that the length of the arc $\kappa$ at $m_{s}$ tends to infinity with $\tilde{\beta}$. If $\alpha_{i} \neq \alpha_{j}$ then $2 l_{\kappa}+l_{\tilde{\alpha}_{i}}+l_{\tilde{\alpha}_{j}}>l_{\tilde{\beta}}$. The lengths of the boundary curves $\tilde{\alpha}_{i}, \tilde{\alpha}_{j}$ are bounded above (and below); since $l_{\tilde{\beta}} \rightarrow \infty$, this forces $l_{\kappa} \rightarrow \infty$. A similar proof works if $\alpha_{i}=\alpha_{j}$.

Finally, we use the hypothesis that $m_{s}$ is the minimum of $F_{s}$ to arrive to a contradiction. The argument is similar to others used above. Let $\gamma_{1}, \ldots, \gamma_{K}$ be simple closed curves extending $\alpha_{1}, \ldots, \alpha_{N}$ to a pants decomposition, and fix a set of dual curves. For each $s$ let $\rho_{s}$ be the surface whose Fenchel-Nielsen coordinates with respect to these choices are

$$
l_{\alpha_{i}}\left(\rho_{s}\right)=l_{\alpha_{i}}\left(m_{s}\right), l_{\gamma_{i}}\left(\rho_{s}\right)=1, t_{\alpha_{i}}\left(\rho_{s}\right)=t_{\gamma_{i}}\left(\rho_{s}\right)=0 .
$$

The surfaces $\rho_{s}$ satisfy the hypothesis of Proposition 4.2, and therefore $l_{\beta_{i}}\left(\rho_{s}\right)=$ $\sum_{j} 2 i\left(\beta_{i}, \alpha_{j}\right) \log \left(1 / l_{\alpha_{j}}\right)+O(1)$. On the other hand, we have

$$
l_{\beta_{i}}\left(m_{s}\right)=\sum_{j} 2 i\left(\beta_{i}, \alpha_{j}\right) \log \left(1 / l_{\alpha_{j}}\right)+f(s),
$$

where $f(s)$ is a positive function which tends to infinity for those curves $\beta_{i}$ intersecting $|\delta|$, since, by the above argument, some arcs of some $\beta_{i}$ outside the collars $A_{j}$ tend to infinity. Thus

$$
F_{s}\left(m_{s}\right)-F_{s}\left(\rho_{s}\right)=s \sum_{i} b_{i}\left(l_{\beta_{i}}\left(m_{s}\right)-l_{\beta_{i}}\left(\rho_{s}\right)\right)=s \sum_{i} b_{i}(f(s)-O(1))
$$

which is positive for small enough $s$.

The following lemma was used in the above proof. We provide a proof, although the result describes a well-known construction.

Lemma 6.3 Let $\rho$ be a hyperbolic surface, let $\alpha_{1}, \alpha_{2}$ be two disjoint simple closed geodesics, and $\bar{\kappa}$ be a geodesic arc from $\alpha_{1}$ to $\alpha_{2}$. Let $\delta$ be a geodesic intersecting $\bar{\kappa}$ and $\tilde{\beta}$ be the 'double' of $\bar{\kappa}$, as constructed in the proof of Proposition 6.2. Then $i(\tilde{\beta}, \delta) \neq 0$. 
Proof Suppose that $\alpha_{1} \neq \alpha_{2}$; by the construction of the curve $\tilde{\beta}$, the curves $\alpha_{1}, \alpha_{2}, \tilde{\beta}$ bound a pair of pants, made up of a thin strip around the arc $\kappa$ (the part of $\bar{\kappa}$ outside the annuli $A_{i}$ ), and the sub-annuli of $A_{1}$ and $A_{2}$ with boundaries $\alpha_{i}$ and $\tilde{\alpha}_{i}$ for $i=1,2$. Correspondingly, there is a pair of pants $P$ in our hyperbolic surface bounded by $\alpha_{1}, \alpha_{2}$ and the geodesic representing $\tilde{\beta}$. Now, $\bar{\kappa}$ is an arc contained in $P$ joining $\alpha_{1}$ to $\alpha_{2}$. The lamination $\delta$ intersects $P$ in arcs which do not meet $\alpha_{1}, \alpha_{2}$, so running from the geodesic representative of $\tilde{\beta}$ to itself; therefore each of these arcs intersects $\bar{\kappa}$ once and $\tilde{\beta}$ twice so that $i(\tilde{\beta}, \delta)=2 i(\bar{\kappa}, \delta)>0$.

In the case that $\alpha_{1}=\alpha_{2}$, remember that the 'double' of $\bar{\kappa}$ is the union of the two simple closed curves $\tilde{\beta}^{\prime}, \tilde{\beta}^{\prime \prime}$ described above. We have to show that $i\left(\tilde{\beta}^{\prime}, \delta\right)+i\left(\tilde{\beta}^{\prime \prime}, \delta\right) \neq 0$. Arguing much as above, we have that $\alpha_{1}$ and the geodesic representatives of $\tilde{\beta}^{\prime}$ and $\tilde{\beta}^{\prime \prime}$ bound a pair of pants containing $\bar{\kappa}$, and $\bar{\kappa}$ joins $\alpha_{1}$ to itself. Since $\delta$ intersects this pair of pants in geodesic arcs not intersecting $\alpha_{1}$, each such arc intersects $\tilde{\beta}^{\prime} \cup \tilde{\beta}^{\prime \prime}$ twice and the result follows.

Finally, we estimate the length along the line of minima of curves dual to the $\alpha_{i}$. Suppose $\alpha_{i}, \gamma_{j}$ is a pants decomposition of the surface $S$, and let $\delta_{i}$ be the dual curves. If $\delta_{i}$ is dual to $\alpha_{i}$, then these two curves intersect either once or twice. If $i\left(\alpha_{i}, \delta_{i}\right)=1$, then $\alpha_{i}$ is on the boundary of just one pair of pants $P$ (two boundary components of $P$ are glued together along $\alpha_{i}$ ). We denote the other boundary component of $P$ by $\omega$. If $i\left(\alpha_{i}, \delta_{i}\right)=2$, then $\alpha_{i}$ is on the boundary of two different pants $P, P^{\prime}$; let $\omega_{1}, \omega_{2}, \omega_{1}^{\prime}, \omega_{2}^{\prime}$ the other boundary components of $P, P^{\prime}$, respectively. To simplify notation, in the following proposition we drop the indices in $\alpha_{i}, \delta_{i}$.

Lemma 6.4 With the above notation, let $\delta$ be the dual curve to $\alpha$.

(a) Suppose that $i(\alpha, \delta)=1$ and that $\rho_{n}$ is a sequence of surfaces so that $l_{\alpha}\left(\rho_{n}\right) \rightarrow 0$ and $\left|t_{\alpha}\left(\rho_{n}\right)\right|$ is bounded. Then

$$
l_{\delta}\left(\rho_{n}\right)=2 i(\alpha, \delta) \log \left(1 / l_{\alpha}\left(\rho_{n}\right)\right)+(1 / 2) l_{\omega}\left(\rho_{n}\right)+O(1) .
$$

(b) Suppose that $i(\alpha, \delta)=2$ and suppose that $\rho_{n}$ is a sequence of surfaces so that $l_{\alpha}\left(\rho_{n}\right) \rightarrow 0$ and $\left|t_{\alpha}\left(\rho_{n}\right)\right|$ is bounded. Then

$$
l_{\delta}\left(\rho_{n}\right)=2 i(\alpha, \delta) \log \left(1 / l_{\alpha}\left(\rho_{n}\right)\right)+l_{|\omega|}\left(\rho_{n}\right)+l_{\left|\omega^{\prime}\right|}\left(\rho_{n}\right)+O(1),
$$

where we denote by $l_{|\omega|}\left(\rho_{n}\right)$ the maximum of $l_{\omega_{1}}\left(\rho_{n}\right)$ and $l_{\omega_{2}}\left(\rho_{n}\right)$, and similarly with $l_{\left|\omega^{\prime}\right|}\left(\rho_{n}\right)$. 
Proof In both cases, the length of $\delta$ depends on the lengths of $\alpha$ and of the neigbouring pants curves and on the twist about $\alpha$. One can calculate $\delta$ explicitly, however it is easier to use the broken arc Lemma 5.1 to simplify the estimates.

If $i(\alpha, \delta)=1$, let $d$ be the distance in $P$ between the two boundary components $\alpha^{\prime}, \alpha^{\prime \prime}$ projecting over $\alpha$. The dual curve $\delta$ can be approximated by a broken arc which wraps part-way round $\alpha^{\prime}$ and then follows the common perpendicular from $\alpha^{\prime}$ to $\alpha^{\prime \prime}$, and finally wraps part-way round $\alpha^{\prime \prime}$. Since we are assuming that $l_{\alpha} \rightarrow 0$ and $\left|t_{\alpha}\left(\rho_{n}\right)\right|$ is bounded, Lemma 5.1 gives the approximation $l_{\delta}=d+O(1)$.

If $i(\alpha, \delta)=2$, let $b, b^{\prime}$ be the lengths of the common perpendicular arcs from $\alpha$ to itself in $P$ and $P^{\prime}$. In this case the approximating broken arc has five segments; three vertical segments which each wrap part-way round arcs which project to $\alpha$, and two horizontal segments which are just the common perpendiculars from $\alpha$ to itself in $P$ and $P^{\prime}$. Thus in this case Lemma 5.1 gives the approximation $l_{\delta}=b+b^{\prime}+O(1)$.

The proof is completed by using the trigonometric formulae in the proof of Lemma 5.4 to estimate $d, b$ and $b^{\prime}$. For (a), if $l_{\omega}$ is bounded above, then $d=2 \log \left(1 / l_{\alpha}\left(\rho_{n}\right)\right)+O(1)$; while if $l_{\omega} \rightarrow \infty$, then $\cosh d \approx e^{l_{\omega} / 2} / l_{\alpha}^{2}$, so the result still holds.

For case (b), note that $P$ is made up of two right-angled hexagons with alternate sides of lengths $l_{\alpha} / 2, l_{\omega_{1}} / 2, l_{\omega_{2}} / 2$ and that $b / 2$ is the distance between $a$ and its opposite side. Let $d_{1}$ be the length of the side between the sides of lengths $l_{\alpha} / 2$ and $l_{\omega_{1}} / 2$. We claim that $b=2 \log \left(1 / l_{\alpha}\right)+l_{|\omega|}+O(1)$, from which, combined with a similar estimate for $b^{\prime}$, part (b) follows.

Since $l_{\alpha} \rightarrow 0$, we have $d_{1} \rightarrow \infty$, and so $\cosh d_{1} \approx \sinh d_{1}$. Thus

$$
\cosh \frac{b}{2} \approx \frac{\cosh \frac{l_{\omega_{2}}}{2}+\cosh \frac{l_{\alpha}}{2} \cosh \frac{l_{\omega_{1}}}{2}}{\sinh \frac{l_{\alpha}}{2} \sinh \frac{l_{\omega_{1}}}{2}} \sinh \frac{l_{\omega_{1}}}{2} \approx \frac{\cosh \frac{l_{\omega_{2}}}{2}+\cosh \frac{l_{\omega_{1}}}{2}}{l_{\alpha}} .
$$

Since $b \rightarrow \infty$, we have $\cosh b / 2 \approx e^{b / 2}$, so by Lemma 5.3 ,

$$
b / 2=\log \left(\cosh \left(l_{\omega_{2}} / 2\right)+\cosh \left(l_{\omega_{1}} / 2\right)\right)+\log \left(1 / l_{\alpha}\right)+O(1) .
$$

Now, expressing $\cosh \left(l_{\omega_{2}} / 2\right)+\cosh \left(l_{\omega_{1}} / 2\right)$ as $\max \left\{\cosh \left(l_{\omega_{2}} / 2\right), \cosh \left(l_{\omega_{1}} / 2\right)\right\}+$ $\min \left\{\cosh \left(l_{\omega_{2}} / 2\right), \cosh \left(l_{\omega_{1}} / 2\right)\right\}$, we easily obtain that

$$
\log \left(\cosh \left(l_{\omega_{2}} / 2\right)+\cosh \left(l_{\omega_{1}} / 2\right)\right)=l_{|\omega|} / 2+O(1) .
$$

Applying this to (6) gives the claim. 
Proposition 6.5 Let $\alpha_{1}, \ldots, \alpha_{N}, \gamma_{1}, \ldots, \gamma_{K}$ be a pants decomposition of $S$ and let $\delta_{i}$ be the dual curve to $\alpha_{i}$. Let $\rho_{n}$ be a sequence so that $l_{\alpha_{i}} \rightarrow 0,\left|t_{\alpha_{i}}\right|$ is bounded and $\rho_{n} \rightarrow\left[a_{1}^{\prime} a_{1}+\cdots+a_{N}^{\prime} \alpha_{N}\right]$. Then, for any $j$ with $a_{j}^{\prime} \neq 0$ we have

$$
l_{\delta_{j}}\left(\rho_{n}\right)=2 i\left(\alpha_{j}, \delta_{j}\right) \log \left(1 / l_{\alpha_{j}}\right)+o\left(\log \left(1 / l_{\alpha_{j}}\right)\right) .
$$

Proof Suppose that $\alpha_{j}, \delta_{j}$ intersect twice (the proof is similar if they intersect once). From Lemma 6.4 (b) we have

$$
l_{\delta_{j}}=2 i\left(\alpha_{j}, \delta_{j}\right) \log \left(1 / l_{\alpha_{j}}\right)+l_{|\omega|}+l_{\left|\omega^{\prime}\right|}+O(1) .
$$

Since $\rho_{n} \rightarrow\left[a_{1}^{\prime} a_{1}+\cdots+a_{N}^{\prime} \alpha_{N}\right]$, we have $l_{\delta_{j}} / c_{n} \rightarrow a_{j}^{\prime} i\left(\alpha_{j}, \delta_{j}\right)$, for some sequence $c_{n} \rightarrow \infty$. If $\omega_{1}$ is one of the curves $\alpha_{i}$, then $l_{\omega_{1}}\left(\rho_{n}\right) \rightarrow 0$. Otherwise, $\omega_{1}$ is one of the curves $\gamma_{i}$, so it is disjoint from the curves $\alpha_{i}$, in which case $l_{\omega_{1}}\left(\rho_{n}\right) / c_{n} \rightarrow i\left(\omega_{1}, a_{1}^{\prime} \alpha_{1}+\cdots+a_{N}^{\prime} \alpha_{N}\right)=0$. The same holds for $\omega_{2}, \omega_{1}^{\prime}, \omega_{2}^{\prime}$. Thus

$$
\lim _{n} \frac{l_{\delta_{j}}\left(\rho_{n}\right)}{c_{n}}=\lim _{n} \frac{2 i\left(\alpha_{j}, \delta_{j}\right) \log \left(1 / l_{\alpha_{j}}\left(\rho_{n}\right)\right)}{c_{n}}=a_{j}^{\prime} i\left(\alpha_{j}, \delta_{j}\right),
$$

which implies that $c_{n} \approx \log \left(1 / l_{\alpha_{j}}\left(\rho_{n}\right)\right)$, and therefore $\frac{l_{|\omega|}\left(\rho_{n}\right)+l_{\left|\omega^{\prime}\right|}\left(\rho_{n}\right)+O(1)}{\log \left(1 / l_{\alpha_{j}}\right)} \rightarrow$ 0 . Thus $l_{|\omega|}\left(\rho_{n}\right)+l_{\left|\omega^{\prime}\right|}\left(\rho_{n}\right)+O(1)=o\left(\log \left(1 / l_{\alpha_{j}}\right)\right)$, which completes the proof.

We can now complete the proof of Theorem 1.1. First, continuing with the assumption that $\nu$ is rational, we follow the method used in Section 4 for the pants decomposition case. Proposition 6.2 shows that the limit of any convergent subsequence of minima $m_{s}$ is a projective lamination $\left[a_{1}^{\prime} \alpha_{1}+\cdots+\right.$ $\left.a_{N}^{\prime} \alpha_{N}\right]$ for some $a_{i}^{\prime} \geq 0$. Proposition 6.1 (c) implies that $\lim \frac{\log \left(1 / l_{\alpha_{i}}\right)}{\log \left(1 / l_{\alpha_{j}}\right)}=1$, for all $i, j$ and therefore, using Proposition 6.5, we argue as in Section 4 to get that $\lim _{s \rightarrow 0} \frac{l_{\delta_{i}}\left(m_{s}\right)}{l_{\delta_{j}}\left(m_{s}\right)}=i\left(\sum_{k} \alpha_{k}, \delta_{i}\right) / i\left(\sum_{k} \alpha_{k}, \delta_{j}\right)$, so that $a_{k}^{\prime}=1$ for all $k$. Thus the limit is independent of the subsequence, and the result follows by compactness of $\operatorname{Teich}(S) \cup \mathrm{P} \mathcal{M L}$. Finally, to complete the proof when $\nu$ is irrational, we follow the outline sketched in Section 5.4.

As a corollary, we obtain an estimate of the length of any closed geodesic along the line of minima $\mathcal{L}_{\mu, \nu}$. This should be compared with the almost identical estimate on p.190 in [9].

Corollary 6.6 Let $\mu=a_{1} \alpha_{1}+\cdots+a_{N} \alpha_{N}$ and $\nu$ be two measured laminations which fill up $S$ (where $\alpha_{1}, \ldots, \alpha_{N}$ is not necessarily a pants decomposition). 
Let $m_{s}$ be the minimum of the function $F_{s}$ and let $\gamma$ be any simple closed curve. Then

$$
l_{\gamma}\left(m_{s}\right)=2 \sum_{j=1}^{N} i\left(\alpha_{j}, \gamma\right) \log \frac{1}{l_{\alpha_{j}}\left(m_{s}\right)}+o\left(\log \frac{1}{s}\right) .
$$

Proof By Theorem 1.1, $m_{s} \rightarrow\left[\sum \alpha_{j}\right]$. This means that

$$
\lim _{s \rightarrow 0} \frac{l_{\gamma}\left(m_{s}\right)}{c_{s}}=i\left(\gamma, \sum \alpha_{j}\right) .
$$

If $\gamma$ is any closed geodesic, then $l_{\gamma}\left(m_{s}\right)=2 \sum i\left(\gamma, \alpha_{j}\right) \log \left(1 / l_{\alpha_{j}}\left(m_{s}\right)\right)+f(s)$, where $f(s)>0$. On the other hand, it is shown in the proof of Proposition 6.5 that

$$
\lim \frac{2 \log \left(1 / l_{\alpha_{j}}\left(m_{s}\right)\right)}{c_{s}}=a_{j}^{\prime}
$$

but we know that $a_{j}^{\prime}=1$. Therefore $\lim \left(f(s) / c_{s}\right)=0$ and the result follows.

\section{References}

[1] A. J. Casson and S. A. Bleiler. Automorphisms of surfaces after Nielsen and Thurston. LMS Lecture Notes 9. Cambridge University Press, 1988.

[2] F. Bonahon. Bouts des variétés de dimension 3. Ann. Math. 124(1), 71-158, 1986.

[3] R. Díaz and C. Series. Examples of pleating varieties for the twice punctured torus. Trans. A.M.S., to appear.

[4] A. Fahti, P. Laudenbach, and V. Poénaru. Travaux de Thurston sur les surfaces, Astérisque 66-67. Société Mathématique de France, 1979.

[5] E. Ghys and P. de la Harpe (eds.). Sur les groupes hyperboliques d'après Mikhael Gromov, Progress in Math. 83. Birkhäuser, 1990.

[6] S. Kerckhoff. Earthquakes are analytic. Comment. Mat. Helv. 60, 17-30, 1985.

[7] S. Kerckhoff. The Nielsen realization problem. Ann. Math. 117(2), 235-265, 1983.

[8] S. Kerckhoff. Lines of Minima in Teichmüller space. Duke Math J. 65, 187-213, 1992.

[9] H. Masur. Two boundaries of Teichmüller space. Duke Math. J. 49, 183-190, 1982.

[10] R. C. Penner with J. Harer. Combinatorics of Train Tracks. Annals of Math. Studies 125. Princeton University Press, 1992. 
[11] J-P. Otal. Le théorème d'hyperbolisation pour les variétés fibrées de dimension 3. Astérisque 235. Société Mathématique de France, 1996.

[12] M. Rees. An alternative approach to the ergodic theory of measured foliations on surfaces. Ergodic Th. and Dyn. Sys. 1, 461-488, 1981.

[13] C. Series. An extension of Wolpert's derivative formula. Pacific J. Math. 197, 223-239, 2000.

[14] C. Series. On Kerckhoff Minima and Pleating Loci for Quasifuchsian Groups. Geometriae Dedicata 88, 211-237, 2001.

[15] C. Series. Limits of quasifuchsian groups with small bending. Warwick preprint, July 2002

[16] W.P. Thurston. The Geometry and Topology of Three-Manifolds. Lecture notes, Princeton University, 1980.

Deparmento Geometría y Topología, Fac. CC. Matemáticas

Universidad Complutense, 28040 Madrid, Spain

and

Mathematics Institute, University of Warwick

Coventry CV4 7AL, UK

Email: radiaz@eucmos.sim.ucm.es and cms@maths.warwick.ac.uk

Received: 17 January 2003

Algebraic 83 Geometric Topology, Volume 3 (2003) 